\title{
Application of Wake Oscillators to Two-Dimensional Vortex-Induced Vibrations of Circular Cylinders in Oscillatory Flows
}

\author{
Pierre-Adrien Opinel \& Narakorn Srinil ${ }^{\dagger}$ \\ Marine, Offshore \& Subsea Technology Group, School of Engineering, Newcastle University, \\ Newcastle upon Tyne, NE1 7RU, United Kingdom
}

\begin{abstract}
A nonlinear time-domain simulation model for predicting two-dimensional vortex-induced vibration (VIV) of a flexibly mounted circular cylinder in planar and oscillatory flow is presented. This model is based on the utilization of van der Pol wake oscillators, being unconventional since wake oscillators have typically been applied to steady flow VIV predictions. The time-varying relative flow-cylinder velocities and accelerations are accounted for in deriving the coupled hydrodynamic lift, drag and inertia forces leading to the cylinder cross-flow and in-line oscillations. The system fluid-structure interaction equations explicitly contain the time-dependent and hybrid trigonometric terms. Depending on the Keulegan-Carpenter number (KC) incorporating the flow maximum velocity and excitation frequency, the model calibration is performed, entailing a set of empirical coefficients and expressions as a function of $\mathrm{KC}$ and mass ratio. Parametric investigations in the case of varying $\mathrm{KC}$, reduced flow velocity, cylinder-to-flow frequency ratio and mass ratio are carried out, capturing some qualitative features of oscillatory flow VIV and exploring the effects of system parameters on response prediction characteristics. The model dependence of hydrodynamic coefficients on the Reynolds number is studied. Discrepancies and limitations versus advantages of the present model with different feasible solution scenarios are illuminated to inform the implementation of wake oscillators as a computationally efficient prediction model for VIV in oscillatory flows.
\end{abstract}

Keywords: vortex-induced vibration (VIV), oscillatory flow, circular cylinder, wake oscillator, KeuleganCarpenter number.

\footnotetext{
† Corresponding author: narakorn.srinil@,newcastle.ac.uk

Tel. +44 191208 6499; Fax +44 1912085491
} 


\section{Nomenclature}

$A_{s}$

$A_{x} / D, A_{y} / D$

$C_{a}$

$C_{D}\left(C_{D 0}\right)$

$C_{L}\left(C_{L 0}\right)$

$C_{D M}$

$C_{M}$

$C_{s}$

$D$

$F_{c x}, F_{c y}$

$F_{D}, F_{L}$

$F_{I}$

$f_{c x}, f_{c y}$

$f_{h}$

$f_{n}$

$f_{r}$

$f_{v}\left(\omega_{v}\right)$

$f_{w}\left(\omega_{w}\right)$

$f_{w x}, f_{w y}$

$f_{x}, f_{y}$

$\mathrm{KC}$

$M_{L}, M_{D}, M_{D M}$

$m_{s}, K_{s}$

$n$

$N_{x}\left(N_{y}\right)$

$p, q$

$\mathrm{Re}$

St

$t(\tilde{t})$

$U$

$U_{m}$

$V_{r}$

$V_{\text {rel }}$

$x, y(X, Y)$

$\alpha_{x}, \alpha_{y}, \beta_{x}, \beta_{y}$

$\alpha_{x}{ }^{*}, \alpha_{y}{ }^{*}, \beta_{x}{ }^{*}, \beta_{y}{ }^{*}$

$\beta$

$\varepsilon_{x}, \varepsilon_{y}\left(\Lambda_{x}, \Lambda_{y}\right)$

$\theta$

$\lambda$

$\mu, m^{*}$

$\xi$

$\rho$

v
Cylinder outer cross-sectional area

In-line and cross-flow amplitudes per diameter

Added mass coefficient of oscillating cylinder in still water

Unsteady drag force coefficient of oscillating (stationary) cylinder

Unsteady lift force coefficient of oscillating (stationary) cylinder

Morison drag force coefficient of stationary cylinder in oscillatory flow

Inertia coefficient of stationary cylinder in oscillatory flow

Cylinder viscous damping coefficient

Cylinder outer diameter

Cylinder feedback terms in dimensional form

Dimensional total drag and lift hydrodynamic forces

Dimensional inertia force

Cylinder feedback terms in dimensionless form

Higher-order nonlinear effect due to relative flow-cylinder velocities

Cylinder natural frequency in still water

Cylinder-to-flow frequency ratio

Fundamental or dominant lift force frequency (angular frequency)

Oscillatory flow frequency (angular frequency)

Total in-line and cross-flow hydrodynamic forces in dimensionless form

Dimensional in-line and cross-flow oscillation frequencies

Keulegan-Carpenter number

Combined hydrodynamic coefficient and mass ratio parameters

Cylinder mass and stiffness in dimensional system

Number of oscillations in the lift force per flow cycle

Number of cylinder vibrations per flow cycle in in-line (cross-flow) direction

Wake oscillator variables modelling the fluctuating drag and lift forces

Reynolds number

Strouhal number

Dimensionless (dimensional) time

Oscillatory flow velocity

Oscillatory flow maximum velocity

Reduced flow velocity parameter

Oscillatory flow-cylinder relative velocities

Dimensionless (dimensional) in-line and cross-flow displacements

Geometrically nonlinear coefficients in dimensionless form

Geometrically nonlinear coefficients in dimensional form

Stokes, viscous or frequency parameter

Empirical wake damping (wake-cylinder coupling) coefficients

Instantaneous angle of lift and drag forces acting on oscillating cylinder

Combined fluid-structural damping terms

Mass parameter and mass ratio

Cylinder damping ratio in still water

Fluid density

Fluid kinematic viscosity 


\section{Introduction}

In coastal, offshore and ocean engineering applications, a cylindrical structure such as a floating spar platform or fixed-foundation monopile supporting wind turbines, an underwater cable, subsea pipeline, marine riser or mooring line may experience vortex-induced vibration (VIV) in oscillatory flow caused by wave or support motion. Although the dynamic features of cylinder VIV in steady flows have been extensively investigated, much less attention has been paid to the modelling and prediction of unsteady and oscillatory flow VIV in the literature. Therefore, practical offshore design in waves is subject to such lacking knowledge and consequent conservatism. With a recent progress in offshore energy development across shallow to deep waters where effects of waves and platform motion become predominant, there is a growing industrial demand for VIV research involving oscillatory flows and a computationally efficient tool for use in a preliminary analysis.

Several theoretical, numerical and experimental studies have contributed to an improved understanding of fluid mechanics, hydrodynamics and wake vortex-shedding visualizations associated with oscillatory flows past stationary cylinders (Justesen, 1991; Obasaju et al., 1988; Sarpkaya, 1976, 1979; Williamson, 1985). One of the key features of a circular cylinder exposed to an oscillatory flow, distinguishing itself from that of a steady flow, is the occurrence of periodic flow reversals causing a return of the shed vortices from being downstream to upstream and a sudden change in the associated hydrodynamic forces with multiple excitation frequencies. These occur whenever the flow velocity changes in sign or direction. As the cylinder is free to move transversely, crossflow VIV may take place with multiple lock-in events leading to multi-peak amplitude response characteristics (Sumer and Fredsoe, 2006). The oscillatory flow VIV behaviour of an elastically mounted circular cylinder is governed by some dimensionless fluid-structure parameters. With a maximum oscillatory flow velocity $U_{m}$, key parameters include the Keulegan-Carpenter number $(\mathrm{KC})$, Reynolds number $(\mathrm{Re})$, reduced flow velocity $\left(V_{r}\right)$ and Strouhal number (St), whose expressions read

$$
\mathrm{KC}=\frac{U_{m}}{f_{w} D}, \quad \operatorname{Re}=\frac{U_{m} D}{v}, V_{r}=\frac{U_{m}}{f_{n} D}, \mathrm{St}=\frac{f_{v} D}{U_{m}},
$$

in which $f_{w}$ is the flow frequency, $D$ the cylinder diameter, $v$ the fluid kinematic viscosity, $f_{v}$ the fundamental or dominant lift force frequency as a result of the vortex shedding and reversal, and $f_{n}$ the structural natural frequency in still water. Equation (1) enables additional relationships:

$$
\frac{\mathrm{KC}}{V_{r}}=\frac{f_{n}}{f_{w}}=f_{r}, \quad \mathrm{StKC}=\frac{f_{v}}{f_{w}}=n, \quad \mathrm{St} V_{r}=\frac{f_{v}}{f_{n}}, \quad \beta=\frac{\mathrm{Re}}{\mathrm{KC}}=\frac{D^{2} f_{w}}{v},
$$

in which $f_{r}$ is the cylinder-to-flow frequency ratio, $n$ is the number of lift force oscillations which may be equivalent to the number of vortex pairs shed from the cylinder plus a unity (i.e. a flow reversal) within each oscillation cycle, and $\beta$ is the so-called Stokes, viscous or frequency parameter (Sarpkaya, 2010). In general, $n$ increases with KC. For stationary cylinders, the oscillatory flow in the $1.8 \times 10^{3}<\operatorname{Re}<10^{4}$ range was found to produce one, two, three and four pairs of vortices for $7<\mathrm{KC}<15,15<\mathrm{KC}<24,24<\mathrm{KC}<32$ and $32<\mathrm{KC}<40$, respectively, by Williamson (1985). Obasaju et al. (1988) showed that one additional vortex is shed per half flow oscillation cycle 
each time $\mathrm{KC}$ is increased by about 8 . This is a consequence of the Strouhal law applicable to an oscillatory flow (Sumer and Fredsoe, 2006).

For a one-degree-of-freedom (1-DOF) cross-flow VIV response, Sumer and Fredsoe (1988) carried out experimental tests using a carriage technique and reported some interesting VIV features of an elastically mounted rigid cylinder in oscillatory flows with $\mathrm{KC}=5,10,20,30,40$ and 100 versus a steady flow case $(\mathrm{KC}=\infty)$, at $\operatorname{Re}=2 \times 10^{4}-10^{5}$. Further, Sumer and Fredsoe (1989) accounted for the Re effect by varying $U_{m}$ and $V_{r}$ from 0 to 16. Therein, $f_{w}$ was also varied to keep KC fixed. For a dominant oscillation frequency of the cylinder $f_{i}$, it may be written that

$$
\frac{f_{i}}{f_{n}}=\frac{f_{i}}{f_{w}}\left(\frac{f_{w}}{f_{n}}\right)=\frac{f_{i}}{f_{w}}\left(\frac{V_{r}}{\mathrm{KC}}\right)=N_{i}\left(\frac{V_{r}}{\mathrm{KC}}\right)=\frac{N_{i}}{f_{r}}
$$

where $N_{i}$ represents the number of cylinder vibrations per flow cycle $\left(N_{i}=f_{i} / f_{w}\right)$ and index $i=x$ or $y$ denotes in-line or cross-flow direction. From Eqs. (2) and (3), $N_{y}=n$ if $f_{y}=f_{v}$ due to a lock-in or resonance. Sumer and Fredsoe (1988) reported that, for $\mathrm{KC}=10, N_{y}=2$ throughout the $V_{r}$ range and the cross-flow amplitude reaches a resonance peak at $f_{y} / f_{n} \approx 1$ at $V_{r}=6$. For $\mathrm{KC}=20$, the response begins with $N_{y}=4$ in a lower $V_{r}$ range, dropping to $N_{y}=3$ and $N_{y}=2$ at $V_{r}=5.5$ and $V_{r}=9$, respectively. This decreased $N_{y}$ is due to the increased $f_{w}$ (for keeping KC fixed) which, in turn, reduces the number of vortices generated per flow cycle (Kozakiewicz et al., 1997). Such a variation of $N_{y}$ entails a multi-peak response occurrence as $V_{r}$ is varied. Similarly, for $\mathrm{KC}=40$, the response starts with $N_{y}=$ 8 , exhibiting a zigzagging trend in the $f_{y} / f_{n}$ plot and consecutively decreasing $N$. This unique multi-peak behaviour is different from a single upper-branch response found in a steady flow VIV (Williamson and Govardhan, 2004). In all $\mathrm{KC}$ cases, $N_{y}=2$ is the absolute minimum number of oscillation cycles. These results suggest that the cylinder cross-flow oscillation pattern follows the fundamental vortex-shedding frequency as $V_{r}$ is increased until reaching a first lock-in point where $N_{y} \approx n\left(f_{y} \approx f_{v}\right)$.

For a 1-DOF inline response prediction, the so-called Morison's equation (Morison et al., 1950) may be used to model an in-line hydrodynamic force acting on a stationary or oscillating cylinder in oscillatory flow. For a stationary cylinder, this semi-empirical equation has two components comprising an inertia force in phase with the local flow acceleration and a drag force proportional to the signed and square of the instantaneous flow velocity. For an oscillating cylinder, the relative velocities and accelerations between the flow and the cylinder are accounted for. The associated inertia and drag coefficients are determined based on experimental data typically depending on KC, Re and surface roughness (Sarpkaya, 2010; Sarpkaya and Isaacson, 1981). Williamson (1985) performed a U-shaped oscillating flow tube test of an elastically mounted cylinder with $\mathrm{KC}$ up to 35 and compared experimental results with those predicted by an extended Morison's equation accounting for the cylinder motion. Near a primary resonance $\left(f_{n} / f_{w} \approx 1\right)$, he suggested that a relative flow-cylinder velocity formulation may be a reasonable assumption for a prediction model. For a fixed $\mathrm{Re}=200$ and $2<\mathrm{KC}<20$, Anagnostopoulos and Iliadis (1998) performed numerical simulations of in-line vibrations of a circular cylinder, capturing the cylinder oscillation effect on the flow pattern and hydrodynamic forces. By varying $f_{w} / f_{n}\left(V_{r} / \mathrm{KC}\right)$ and changing $f_{n}\left(V_{r}\right)$ for a 
fixed $\mathrm{KC}=10$ and 20 , they reported that the in-line force contains $f_{w}$ and the odd multiples of $f_{w}$ components amplifying the cylinder response near resonance.

Experimental and numerical results for 2-DOF in-line and cross-flow responses are very sparse. Bearman and Mackwood (1991) conducted experiments in a U-tube water tank generating oscillatory flows in a range of $0<\mathrm{KC}<50$ and maximum $\beta$ of $750\left(\mathrm{Re}=3.75 \times 10^{4}\right)$. Their results emphasize the cross-flow VIV dependence on $\mathrm{KC}$ and $V_{r}$ (i.e. $\left.f_{n} / f_{w}\right)$, justifying the applicability of Eq. (3). The in-line response increases monotonically with $\mathrm{KC}$ such that $f_{x} \approx f_{w}$, regardless of the cross-flow motion being restrained or allowed. In some cases, the in-line response may be modified due to the cross-flow oscillation. This two-directional fluid-cylinder interaction and coupling were further experimentally investigated by Lipsett and Williamson (1994) who measured cross-flow $(Y)$ and inline $(X)$ cylinder responses in oscillatory flows with $2<\mathrm{KC}<60$ and $1<f_{n} / f_{w}<9$. At low $f_{n} / f_{w}<4$, repeatable $X$-Y trajectories with variable phase differences were captured depending on both $\mathrm{KC}$ and $f_{n} / f_{w}$, with $X$ responses being greater than $Y$ responses. At higher $f_{n} / f_{w}$, the trajectories become more irregularly complex due to the increasing transverse cycles of each in-line periodic oscillation. While the in-line maximum response increases with $\mathrm{KC}$, the cross-flow response remains comparable to that in a lower $f_{n} / f_{w}$ case. More recently, VIV phenomena in oscillatory flows have further been captured through two-dimensional numerical simulations by Zhao (2013) who considered a flexibly mounted circular cylinder in oscillatory flows with a fixed $\mathrm{KC}=10,20$ and 40, 308 $<\mathrm{Re}<9240$ and $0<V_{r}<30$. For a given $\mathrm{KC}$, both $U_{m}$ and $f_{w}$ were simultaneously varied. Zhao (2013) showed that cross-flow VIV exhibits a multi-peak response subject to multiple excitation frequencies with a primary component decreasing as $V_{r}$ is increased. When the response is regular and repeatable, $N_{y}$ is found to be equal to the number of vortex pairs shed per each cycle plus 1 associated with a flow reversal event. This implies that, for a lock-in, $N_{y} \approx n$ as in 1DOF cross-flow VIV studies. Nevertheless, at a transition boundary between two different response regimes, the vibration trajectories become irregular. In agreement with Williamson (1985), Bearman and Mackwood (1991), the primary frequency of in-line response is equal to $f_{w}$ for all $\mathrm{KC}$ and $V_{r}$. Zhao (2013) also reported that the inline amplitude becomes significantly greater than the cross-flow one as $\mathrm{KC}$ and/or $V_{r}$ is increased, with the maximum value of about $6 D$ for $\mathrm{KC}=40$. This is much larger than cross-flow and in-line VIV amplitudes in steady flows for the same mass-damping cylinder. It is important to remark that the multi-frequency feature of cross-flow VIV in oscillatory flows would play a meaningful role in fatigue life analysis of offshore structures in waves.

Recently, a few researchers have investigated VIV of long flexible cylinders in oscillatory flows. Fu et al. (2014) performed experimental tests and reported cross-flow VIV of a horizontal cylinder whose ends were forced to harmonically move in still water for $26<\mathrm{KC}<178$ and for specific $V_{r}=4,6.5$ and 7.9. Depending on $\mathrm{KC}$, they suggested three regions including the building-up, lock-in and dying-out cross-flow VIV. Key features of amplitude modulation, modal transition, hysteresis and intermittent response were remarked. For $\mathrm{KC}=84$, Fu et al. (2018) carried out numerical simulations using a strip theory for the tested cylinder model of Fu et al. (2014). They reported the occurrence of such a three-stage VIV development process and hysteresis between flow decelerating and accelerating events in oscillatory flows. Similar time-varying characteristics were reported by Wang et al. 
(2018) for VIV of a free-hanging catenary riser subject to a vessel motion-induced oscillatory flow. Nevertheless, these experimental and numerical results are based on an imposed end excitation. These observations might not be directly applicable to the oscillatory flow-induced VIV of flexible cylinders with fixed ends.

From a modelling and prediction viewpoint, there have been just a few attempts in the literature to semiempirically model coupled in-line and cross-flow hydrodynamic forces acting on cylinders in oscillatory flows. The Morison's equation is commonly used to describe an in-line force (Lipsett and Williamson, 1994; Williamson, 1985). Bearman et al. (1984) proposed a quasi-steady model to predict the transverse forces on cylinders in waves and oscillatory flows. This model assumes $\mathrm{St}=0.2$ and a constant amplitude of the lift coefficient over a half flow cycle, providing a good prediction when compared with experimental results for $15<\mathrm{KC}<53$. Thorsen et al. (2016) presented a transverse force model including the excitation, damping and added mass terms. The lift excitation coefficient depends on the response amplitude, and the force fluctuation depends on the time-varying phase difference between the cylinder motion and force excitation. The damping term is nonlinear, depending on the cylinder velocity and the drag coefficient as a function of cylinder response. Some empirical coefficients were proposed and limited to the available low-amplitude data range. This model has recently been extended to account for in-line VIV in oscillatory flows by Ulveseter et al. (2018).

As an alternative approach, Hayashi (1984) was perhaps the first who attempted to apply a van der Pol wake oscillator, developed by Hartlen and Currie (1970) for steady flows, to model the fluctuating lift forces in oscillatory flows in predicting cross-flow VIV in waves. The amplification of the lift force around a resonance was reproduced, but the model was unable to predict the cylinder response in a certain frequency ratio range. In addition, the effect of in-line motion was neglected. To the present authors' knowledge, the application of nonlinear wake oscillators to predict oscillatory flow VIV is still lacking in the literature. Therefore, it is the main aim of the present study to investigate the feasibility and limitation of using wake oscillators for modelling combined cross-flow/in-line VIV in oscillatory flows, by extending previous model developments and applications in steady flow VIV cases for both rigid (Bai and Qin, 2014; Facchinetti et al., 2004; Srinil et al., 2018; Zanganeh and Srinil, 2014) and long flexible (Gao et al., 2019; Low and Srinil, 2016; Srinil, 2010, 2011; Srinil et al., 2009; Xu et al., 2008; Yang et al., 2018; Zanganeh and Srinil, 2016) cylinders.

This paper is structured as follows. In Section 2, the mathematical equations of coupled cross-flow and inline motions describing the nonlinear fluid-cylinder interaction of a 2-DOF VIV in oscillatory flow are presented. The forcing terms, a set of dimensionless parameters and empirical coefficients are defined. The model calibration and sensitivity studies are carried out in Section 3 introducing a new set of empirical coefficients and functions depending on $\mathrm{KC}$ and mass ratio. In Section 4, parametric studies are performed with different model solution scenarios to highlight several 2-DOF VIV prediction characteristics, maximum response amplitudes, oscillation frequencies and effects of system parameters including $\mathrm{KC}, V_{r}$, system frequency ratios and mass ratio. The paper ends with conclusions in Section 5. 


\section{Oscillatory Flow VIV Prediction Model}

A mathematical model to predict the fluid-structure interaction for VIV of a rigid circular cylinder in a planar and oscillatory flow is presented by utilizing a nonlinear wake oscillator model recently advanced in Srinil et al. (2018) following an original concept in Srinil and Zanganeh (2012) to account for three main aspects. These include (i) the effect of nonlinear coupling of cross-flow and in-line responses as well as lift and drag hydrodynamic forces, (ii) a resonance range capturing a self-limiting peak response when varying flow velocities, and (iii) the interrelationships of empirical coefficients and system fluid-structure parameters enabling the associated function applicability to a wide parametric range. Such a reduced-order model has been satisfactorily used for predicting steady flow VIV of long flexible cylinders with multi DOF responses (Zanganeh and Srinil, 2016).

Figure 1 displays a two-dimensional spring idealization of a flexibly mounted circular cylinder horizontally placed in a uniform planar sinusoidal flow whose the time-varying horizontal velocity function $U$ is assumed as

$$
U=U_{m} \sin \left(\omega_{w} \tilde{t}\right)
$$

where $\omega_{w}$ is the angular frequency of the oscillatory flow $\left(\omega_{w}=2 \pi f_{\mathrm{w}}\right)$ and $\tilde{t}$ denotes the dimensional time. This flow function has been widely used as the most common assumption in experimental (Lipsett and Williamson, 1994; Sumer and Fredsoe, 2006) and numerical (Zhao, 2013) studies. A direction of the instantaneous relative velocity of the incoming flow with respect to the cylinder $\left(V_{\text {rel }}\right)$ is described by a dynamic angle $\theta$ representing an effective direction of fluctuating drag (i.e. aligned with $V_{r e l}$ ) and lift (perpendicular to $V_{r e l)}$ forces. Accordingly,

$$
V_{\text {rel }}=\sqrt{(U-\dot{X})^{2}+\dot{Y}^{2}}
$$

in which a dot denotes differentiation with respect to $\tilde{t}$. In this study, the total drag $F_{D}$ and lift $F_{L}$ hydrodynamic forces per unit length may be expressed as

$$
F_{D}=\frac{1}{2} \rho D V_{r e l}^{2}\left(C_{D M}+C_{D}\right), \quad F_{L}=\frac{1}{2} \rho D V_{r e l}^{2} C_{L},
$$

where $\rho$ is the fluid density, $C_{D}$ and $C_{L}$ are the fluctuating drag and lift coefficients associated with the vortex shedding, and $C_{D M}$ is the Morison drag coefficient obtained from a stationary cylinder in an oscillatory flow. The constant $C_{D M}$ is dependent on KC and Re (Sarpkaya and Isaacson, 1981) whose the forcing effect is, nevertheless, time-dependent and may be amplified by $V_{r e l}$. The amplitudes of $C_{D}$ and $C_{L}$ may be modulated as the cylinder oscillates, depending on response amplitudes, frequencies and fluid-structure parameters. Such features will be accounted for by the wake oscillators. If the vortex shedding, lift force and transverse response are neglected, the combination of Eq. (5) and the first expression in Eq. (6) entails the Morison drag force $\rho D C_{D M}(U-\dot{X})|U-\dot{X}| / 2$ accounting for the body oscillation (Sumer and Fredsoe, 2006). Note that Lipsett and Williamson (1994) also presented a numerical fluid model as in Eq. (6), but they neglected $C_{D}$ from their model and further postulated the lift coefficient variation through a harmonic function with a predefined phase difference between the lift force and the flow, despite the fact that such a relative phase is unknown in practice. 
Following a mathematical derivation of a two-directional spring-mass system described in the Appendix of Srinil et al. (2018), $F_{L}$ and $F_{D}$ may be projected onto the $X$ and $Y$ coordinates as $F_{L} \sin \theta+F_{D} \cos \theta$ and $F_{L} \cos \theta$ $F_{D} \sin \theta$, respectively, with actual $\sin \theta=\dot{Y} / V_{r e l}$ and $\cos \theta=(U-\dot{X}) / V_{r e l}$ relations. The acceleration force in the $X$ direction consists of two components: one being associated with the pressure gradient (Froude-Krylov) causing the fluid acceleration $(\dot{U})$ independent of the cylinder motion, and the other depending on the cylinder acceleration relative to the flow $(\ddot{X}-\dot{U})$. The acceleration force in the $Y$ direction only accounts for the latter component $(\ddot{Y})$. As a result, the direction of the cylinder acceleration with respect to the flow is not necessarily the same as $V_{\text {rel }}$ direction, depending on the magnitude of $\sqrt{(\ddot{X}-\dot{U})^{2}+\ddot{Y}^{2}}$. In the same way as the projection of lift and drag forces, the $X$ and $Y$ acceleration forces may be resolved and approximated as $C_{M} \rho A_{s} \dot{U}-C_{a} \rho A_{s} \ddot{X}$ and $-C_{a} \rho A_{s} \ddot{Y}$, respectively (Williamson, 1991). Consequently, by combining all the hydrodynamic force components, the geometrically nonlinear coupled oscillators governing in-line and cross-flow vibrations of an elastically mounted circular cylinder in an oscillatory flow may be expressed in a dimensional form as

$$
\begin{aligned}
& m_{s} \ddot{X}+C_{s} \dot{X}+K_{s}\left(X+\alpha_{x}^{*} X^{3}+\beta_{x}^{*} X Y^{2}\right)=F_{L} \frac{\dot{Y}}{V_{\text {rel }}}+F_{D} \frac{(U-\dot{X})}{V_{r e l}}+C_{M} \rho A_{s} \dot{U}-C_{a} \rho A_{s} \ddot{X}, \\
& m_{s} \ddot{Y}+C_{s} \dot{Y}+K_{s}\left(Y+\alpha_{y}^{*} Y^{3}+\beta_{y}^{*} Y X^{2}\right)=F_{L} \frac{(U-\dot{X})}{V_{r e l}}-F_{D} \frac{\dot{Y}}{V_{r e l}}-C_{a} \rho A_{s} \ddot{Y},
\end{aligned}
$$

where $m_{s}, A_{s}, C_{s}$ and $K_{s}$ are the structural mass, outer cross-sectional area, viscous damping and spring stiffness coefficients, respectively, and $C_{a}$ is the added mass coefficient of the oscillating cylinder in still water $\left(C_{a}=1\right.$ for a circular cylinder according to Blevins (1990)). The inertia force $F_{I}$ is $C_{M} \rho A_{s} \dot{U}$ in which $C_{M}$ is the inertia coefficient of the stationary cylinder in oscillatory flow $\left(C_{M} \neq C_{a}+1\right)$. The actual inertia or added mass coefficient of the oscillating cylinder in oscillatory flow is unknown a priori, depending on the response amplitude, frequency, relative motion and VIV effect. Herein, $C_{M}$ is considered, which is dependent on KC and Re (Sarpkaya, 2010). Both $C_{a}$ and $C_{M}$ assumptions have been applied by Lipsett and Williamson (1994). If the cylinder is stationary in an oscillatory flow, the Morison's equation, comprising the in-line drag and inertia forces in the absence of vortex shedding, is $\rho D C_{D M} U|U| / 2+\rho A_{s} C_{M} \dot{U}$ (Morison et al., 1950). According to Srinil et al. (2018), the geometrically nonlinear coefficients $\alpha_{x}^{*}, \beta_{x}^{*}, \alpha_{y}^{*}$ and $\beta_{y}^{*}$ are functions of the spring length. These arbitrary quantities may be treated as empirically tuned coefficients to capture relevant VIV features. The effects of $C_{D M}$ and $C_{M}$ on the prediction will be discussed via model calibration in Section 3.

With respect to the modelling of the vortex-shedding effect, $C_{D}$ and $C_{L}$ fluctuations may be represented through the wake oscillator variables $p=2 C_{D} / C_{D 0}$ (Srinil and Zanganeh, 2012) and $q=2 C_{L} / C_{L 0}$ ((Facchinetti et al., 2004), in which $C_{D 0}$ and $C_{L 0}$ are the associated oscillating drag and lift coefficients of a stationary cylinder in oscillatory flow. The time variation of $p$ and $q$ may be modelled through the van der Pol wake oscillators as

$$
\ddot{p}+\varepsilon_{x} \omega_{v}\left(p^{2}-1\right) \dot{p}+\omega_{v}^{2} p=F_{c x},
$$




$$
\ddot{q}+\varepsilon_{y} \omega_{v}\left(q^{2}-1\right) \dot{q}+\omega_{v}^{2} q=F_{c y},
$$

in which $\varepsilon_{x}$ and $\varepsilon_{y}$ are the empirical damping coefficients, $F_{c x}$ and $F_{c y}$ are the cylinder (displacement, velocity or acceleration) feedback terms simulating the fluid-cylinder interaction effect, and $\omega_{v}$ is the fundamental angular frequency of the lift force associated with the vortex shedding $\left(\omega_{v}=2 \pi f_{v}\right)$. Herein, $\omega_{v}$ is applied to both the drag (Eq. 9) and lift (Eq. 10) force oscillators since recent numerical simulations of 2-DOF VIV in oscillatory flows by Zhao (2013) suggest a common primary response frequency in both cross-flow and in-line directions, in addition to the flow frequency $\left(f_{w}\right)$. This feature is different from a steady flow VIV case where a 2:1 (drag-lift) frequency ratio has been applied to the wake oscillators simulating a figure-of-eight $X$ - $Y$ trajectory (Srinil and Zanganeh, 2012). In addition to $f_{v}$ and $f_{w}$, the higher-order frequency components in both $x$ - $y$ responses have been observed by Zhao (2013) as well as Lipsett and Williamson (1994). By accounting for the relative velocities between the flow and the cylinder, such an appearance of multi-frequency response is accounted for through the multiplication of $f_{v}$-dependent $(p, q)$ and $f_{w}$-dependent ( $U$ ) variables (i.e. $F_{L}$ and $F_{D}$ terms) in Eqs. (7) and (8). By assigning $f_{v}$ equivalently in both drag-lift oscillators, the in-line response frequency would contain the primary component $f_{\mathrm{w}}$ (due to oscillatory flow) and the high-order components (due to VIV), one of which potentially matching the dominant cross-flow response frequency, depending on $\mathrm{KC}$ and $V_{r}$. If a higher-order excitation frequency (e.g. $2 f_{v}$ ) is specified in the drag wake oscillator, the in-line response would respond with higher-order frequencies considerably differing from those primarily governing the cross-flow response, due to such multiplied and nonlinear terms.

By introducing the dimensionless time $\left(t=\omega_{w} \tilde{t}\right)$ and displacements $(x=X / D, y=Y / D)$, incorporating the wake oscillator $(p, q)$ variables into Eqs. (6)-(8), and accounting for the relationships in Eq. (1), the system of nonlinear equations of coupled motions of the elastically mounted rigid circular cylinder undergoing 2-DOF VIV under oscillatory flow may be expressed in a dimensionless form as

$$
\begin{aligned}
& \ddot{x}+\lambda(\dot{x}, \dot{y}, t) \dot{x}+\left(\mathrm{KC} / V_{r}\right)^{2}\left(x+\alpha_{x} x^{3}+\beta_{x} x y^{2}\right)=f_{w x}(\dot{x}, \dot{y}, t), \\
& \ddot{p}+\varepsilon_{x} \operatorname{StKC}\left(p^{2}-1\right) \dot{p}+(\mathrm{StKC})^{2} p=f_{c x}, \\
& \ddot{y}+\lambda(\dot{x}, \dot{y}, t) \dot{y}+\left(\mathrm{KC} / V_{r}\right)^{2}\left(y+\alpha_{y} y^{3}+\beta_{y} y x^{2}\right)=f_{w y}(\dot{x}, \dot{y}, t), \\
& \ddot{q}+\varepsilon_{y} \operatorname{StKC}\left(q^{2}-1\right) \dot{q}+(\operatorname{StKC})^{2} q=f_{c y},
\end{aligned}
$$

where a dot now denotes differentiation with respect to $t, \alpha_{x}^{*}, \beta_{x}^{*}, \alpha_{y}^{*}$ and $\beta_{y}^{*}$ become the dimensionless (dependent on a ratio of $D$ to the spring length) and empirical variables $\alpha_{x}, \beta_{x}, \alpha_{y}$ and $\beta_{y}, f_{c x}=F_{c x} / D \omega_{w}^{2}$ and $f_{c y}=F_{c y} / D \omega_{w}^{2}$. Following Facchinetti et al. (2004) for a steady flow VIV, one may specify $f_{c x}=\Lambda_{x} x, f_{c x}=\Lambda_{x} \dot{x}$ or $f_{c x}=\Lambda_{x} \ddot{x}$ ( $f_{c y}=\Lambda_{y} y, f_{c y}=\Lambda_{y} \dot{y}$ or $\left.f_{c y}=\Lambda_{y} \ddot{y}\right)$ as a first-order linear in-line (cross-flow) displacement, velocity or acceleration coupling, respectively, with empirical coefficients $\Lambda_{x}$ and $\Lambda_{y}$ to be identified together with $\varepsilon_{x}$ and $\varepsilon_{y}$. The fluctuating in-line $\left(f_{w x}\right)$ and cross-flow $\left(f_{w y}\right)$ hydrodynamic forces read 


$$
\begin{aligned}
& f_{w x}(\dot{x}, \dot{y}, t)=\left(2 \pi M_{L} q \dot{y}+M_{D M} \mathrm{KC} \sin (t)+M_{D} p(\mathrm{KC} \sin (t)-2 \pi \dot{x})\right) f_{h}(\dot{x}, \dot{y}, t)+C_{M} \frac{\mathrm{KC}}{8 \mu} \cos (t), \\
& f_{w y}(\dot{x}, \dot{y}, t)=\left(M_{L} q(\mathrm{KC} \sin (t)-2 \pi \dot{x})-2 \pi M_{D} p \dot{y}\right) f_{h}(\dot{x}, \dot{y}, t) .
\end{aligned}
$$

The dimensionless quantities $\left(M_{L}, M_{D}, M_{D M}\right)$, the mass parameter $(\mu)$, the time-varying damping $(\lambda)$ and the higher-order nonlinear effect of the relative velocities $f_{h}(\dot{x}, \dot{y}, t)$ are defined, respectively, as

$$
\begin{gathered}
M_{L}=\frac{C_{L 0}}{16 \pi^{2} \mu}, \quad M_{D}=\frac{C_{D 0}}{16 \pi^{2} \mu}, \quad M_{D M}=\frac{C_{D M}}{8 \pi^{2} \mu}, \quad \mu=\pi\left(m^{*}+C_{a}\right) / 4, \\
\lambda(\dot{x}, \dot{y}, t)=2 \xi\left(\frac{\mathrm{KC}}{V_{r}}\right)+\frac{C_{D M}}{4 \pi \mu} f_{h}(\dot{x}, \dot{y}, t), \\
f_{h}(\dot{x}, \dot{y}, t)=\sqrt{(\mathrm{KC} \sin (t)-2 \pi \dot{x})^{2}+(2 \pi \dot{y})^{2}},
\end{gathered}
$$

where $\xi=C_{s} / 2 \sqrt{K_{s}\left(m_{s}+C_{a} \rho A_{s}\right)}$ and $m^{*}$ is the mass ratio with $m^{*}=4 m_{s} / \rho \pi D^{2}$. As $C_{a}=1$ is herein assumed for a circular cylinder, $\xi$ is equivalent to the cylinder damping ratio in still water. In both $X$ - $Y$ directions, $\xi, m^{*}$ and $f_{n}$ are assumed to be constants. It is worth remarking that both Eqs. (11) and (13) are highly nonlinear and nonhomogeneous (explicitly time-dependent), containing a mixture of cosine and sine functions in the $X$ direction. A multiplication of sine terms in Eqs. (15) and (16) potentially leads to multiple competing frequencies. If the flow is steady and uniform (i.e. $U$ is a constant), the system of equations of 2-DOF motions presented in Srinil et al. (2018) will be recovered for steady flow VIV.

Based on Eq. (2), StKC $=n$ in Eqs. (12) and (14). For stationary cylinders, Sarpkaya (1976) suggested that $n=2,4$ and 8 for $\mathrm{KC}=10,20$ and 40, respectively, enabling $\mathrm{St}=0.2$. Sarpkaya (2010) further noted that the lift and drag forces become increasingly important as $\mathrm{KC}>5$ and 15 , respectively, whereas the inertia force dominates when KC $<10$. For 1-DOF cross-flow VIV in oscillatory flows, Sumer and Fredsoe (1988) suggested, based on Eq. (3), that $N_{y}=2,4-5$ and 8-10 for $\mathrm{KC}=10,20$ and 40, respectively, signifying a similar trend of $n$ and $N_{y}$ and a variable $N_{y}$ at a higher KC for the oscillating cylinder. For 2-DOF VIV, Zhao (2013) reported, through 2-D numerical simulations, the dependence of $N_{y}$ on both $\mathrm{KC}$ and $V_{r}$ when the latter is varied. For $\mathrm{KC}=10, N_{y}=2$ in the range of $V_{r}=5-13$. For $\mathrm{KC}=20, N_{y}=5$ at $V_{r}=4, N_{y}=4$ at $V_{r}=5$ and $N_{y}=3$ at $V_{r}=8-13$. For $\mathrm{KC}=40, N_{y}=8$ at $V_{r}=5, N_{y}=7$ at $V_{r}=6, N_{y}=6$ at $V_{r}=8, N_{y}=5$ at $V_{r}=10-20$ and $N_{y}=4$ at $V_{r}=26-30$. The $x-y$ trajectories associated with these $V_{r}$ are repeatable whereas they become quite chaotically irregular during the $N_{y}$ mode transition. The effects of specifying $n$ and empirical coefficients on VIV prediction will be discussed in Section 3 . The highly nonlinear Eqs. (11)-(14) can be numerically integrated using a fourth-order Runge-Kutta scheme with an adaptive time step, and $x=y=\dot{x}=\dot{y}=\dot{p}=\dot{q}=0, p=2$ and $q=2$ as initial conditions. Steady-state responses of about 80 dimensionless flow cycles will be analyzed to present cylinder response amplitudes and frequencies.

\section{Calibration and Selection of Empirical Coefficients}

For the system of Eqs. (11)-(17), there are several empirical coefficients to be identified among the hydrodynamics 
$\left(C_{M}, C_{D M}, C_{a}, C_{L 0}, C_{D 0}, \mathrm{St}\right)$, structure $\left(\alpha_{x}, \beta_{x}, \alpha_{y}, \beta_{y}\right)$ and wake oscillator $\left(\varepsilon_{x}, \Lambda_{x}, \varepsilon_{y}, \Lambda_{y}\right)$ coefficients, depending on the key input fluid-structure parameters $\left(m^{*}, \xi, \mathrm{KC}, V_{r}, \mathrm{Re}\right)$. Recall also that $\mathrm{StKC}$ may be equivalent to $n$ and specified a priori; alternatively, St and KC may be individually specified, with the latter being varied so that a resonance between the two sets of oscillators may be achieved. For the present wake-cylinder oscillators, it would be impractical to determine a universal set of empirically tuned coefficients (up to 14 variables) for predicting any cylinder VIV in oscillatory flow, considering that experimental data in 2-DOF VIV cases are still scarce. For model calibration, numerical results from the computational fluid dynamics (CFD) studies of Zhao (2013) are herein considered for 2-DOF VIV of a circular cylinder subject to a two-dimensional planar oscillatory flow with $\mathrm{KC}=10,20$ and 40. In such CFD work, $m^{*}=1.62, \xi=0.012$ and $\operatorname{Re} / V_{r}=308$, and the associated Re was varied from 308 to 9240 in the range of $1<V_{r}<30$ in which $U_{m}$ and $f_{w}$ were simultaneously varied for a constant KC, $f_{n}$ and $D$. These parameters and scenarios will be considered as model inputs in the following simulations, unless stated otherwise.

Based on preliminary sensitivity studies as in Srinil et al. (2018) and calibration of $x-y$ response amplitudes versus CFD results of Zhao (2013), it has been deduced that $\alpha_{x}$ and $\beta_{y}$ play the most influential role in predicting 2-DOF VIV in oscillatory flows. This is because $\alpha_{x}$ and $\beta_{y}$ govern the in-line displacement affecting the nonlinear stiffness $x^{3}$ term in Eq. (11) for the in-line direction and $y x^{2}$ term in Eq. (13) for the cross-flow direction. The inline amplitude increases and becomes much greater than the cross-flow counterpart as $\mathrm{KC}$ and/or $V_{r}$ increases (Zhao, 2013). Hence, $\alpha_{x}$ and $\beta_{y}$ may be specifically tuned for amplitude calibration purpose. As for the wakecylinder coupling terms in Eqs. (12) and (14), $f_{c x}=\Lambda_{x} \ddot{x}$ and $f_{c y}=\Lambda_{y} \ddot{y}$ may be applied as in the steady flow VIV case (Facchinetti et al. 2004), see also discussion later on. Herein, based on $308<\operatorname{Re}<9240$ (Zhao 2013), we specify $C_{M}=1, C_{D M}=2$ and $C_{a}=1$ (Sarpkaya, 2010). Following Srinil et al. (2018), $C_{L 0}=0.3, C_{D 0}=0.2, \beta_{x}=\alpha_{y}=$ $0.7, \varepsilon_{x}=0.3, \Lambda_{x}=12$ and $\varepsilon_{y}=0.00234 \mathrm{e}^{0.228 \mathrm{~m}^{*}}$ whereas $\Lambda_{y}=38$ based on the present calibration which accounts for the tuning of the two most influential variables $\alpha_{x}$ and $\beta_{y}$ in order to predict cross-flow and in-line amplitudes and associated qualitative features (including a multi-peak cross-flow response and a monotonically increasing in-line response) as $V_{r}$ is increased for the assigned input parameters ( $\left.n, \mathrm{KC}, m^{*}, \xi\right)$. A MATLAB solver (e.g. 'fminsearch') has been applied to minimize the error function (i.e. the root-mean-square deviation of the wake oscillator predictions from the associated CFD results), and to optimize the obtained results based on solving the system of Eqs. (11)-(17) with multiple variables. This calibration has enabled us to identify a single set of best-fit empirical variables for both wake-cylinder oscillators applicable to the whole considered $V_{r}$ range. Consequently, new empirical expressions capturing the $\mathrm{KC}$ and $m^{*}$ dependence may be introduced as

$$
\begin{aligned}
& \alpha_{x}=0.46 \mathrm{e}^{-0.082 \mathrm{KC}}, \\
& \beta_{y}=2.22 \mathrm{e}^{-0.032 \mathrm{KC}} \times 1.416 \mathrm{e}^{-0.215 m^{*}}=3.143 \mathrm{e}^{-\left(0.032 \mathrm{KC}+0.215 m^{*}\right)} .
\end{aligned}
$$

As KC or $m^{*}$ is increased, $\alpha_{x}$ and $\beta_{y}$ decrease through the above expressions, diminishing the effect of cylinder nonlinear stiffness, because the resulting values of the associated $\alpha_{x} x^{3}$ (Eq. 11) and $\beta_{y} y x^{2}$ (Eq. 13) terms decrease. 
The associated trend of $\alpha_{x}$ or $\beta_{y}$ as a function of $\mathrm{KC}$, as well as $\beta_{y}$ as a function of $m^{*}$, is shown in Fig. 2a whereas a contour plot of $\beta_{y}$ as function of both $\mathrm{KC}$ and $m^{*}$ is shown in Fig. 2b. A decrease of $\alpha_{x}$ would yield a greater inline response whereas a decrease of $\beta_{y}$ would yield a decrease of cross-flow response particularly in a high $V_{r}$ range $(>15)$ as this would be expected for a higher $m^{*}$ for 2-DOF VIV. If Eq. (19) is only dependent on KC, the predicted cross-flow response will become qualitatively erroneous when varying $m^{*}$.

Figure 3 compares $x-y$ response amplitudes $\left(A_{x} / D, A_{y} / D\right)$ based on the wake oscillator and CFD (Zhao, 2013) models, by also accounting for the effect of specified $n$. For $\mathrm{KC}=10,20$ and 40, $n$ might be equal to 2,4 and 8 (Sarpkaya, 1976) and specified in the range of 2-4, 4-6 and 8-10, respectively, according to an observation of oscillatory flow around the stationary cylinder in the literature (Sumer and Fredsoe, 2006). For in-line responses, CFD results in Fig. 3 show that the maximum $A_{x} / D$ occurs at a higher $V_{r}$ as $\mathrm{KC}$ is increased. At $\mathrm{KC}=10, A_{x} / D$ response (Fig. 3a) reveals a resonance peak at $V_{r} \approx 13-14$ captured by both models despite having different maximum amplitudes. At $\mathrm{KC}=20, \mathrm{CFD}$ results of $A_{x} / D$ (Fig. 3c) reveal a peculiar peak at $V_{r} \approx 18-19$ which may be due to the coupling with $A_{y} / D$ (Fig. 3d). However, this feature was not reported in Zhao (2013). The wake oscillator model does not admit such a peak but predicts how $A_{x} / D$ increases with $V_{r}$ as in the CFD study and other experimental tests (Williamson, 1985; Bearman and Mackwood, 1991). Greater amplitude differences are noticed in Fig. 3c. Any other tuning based on a trial of different sets of empirical wake oscillator variables $\left(\varepsilon_{x}, \Lambda_{x}, \varepsilon_{y}, \Lambda_{y}\right)$ was unsatisfactory for this $\mathrm{KC}=20$, since an error function could not be minimized during calibration to achieve simultaneously the coupled in-line and cross-flow response predictions. Nevertheless, such $A_{x} / D$ discrepancies at $\mathrm{KC}=10$ and 20 are reduced when increasing $\mathrm{KC}$ to 40 as shown in Fig. 3e exhibiting a monotonic trend and greater response as $V_{r}$ is increased $\left(A_{x} / D \approx 6\right.$ at $\left.V_{r}=30\right)$. While $A_{x} / D$ values predicted by wake oscillators are slightly sensitive to the $n$ change at lower $\mathrm{KC}=10$ and 20, the predicted $A_{x} / D$ becomes more sensitive at higher $\mathrm{KC}=40$ for $V_{r}<18$.

For cross-flow responses, CFD results in Fig. $3 \mathrm{~b}(\mathrm{KC}=10), \mathrm{d}(\mathrm{KC}=20)$ and $\mathrm{f}(\mathrm{KC}=40)$ show that $A_{y} / D$ responses relatively fluctuate, exhibiting multiple peaks $\left(A_{y} / D \approx 0.7-1\right)$ as $V_{r}$ is increased, and suggesting several lock-in events. At low $\mathrm{KC}=10, A_{y} / D$ significantly diminishes for $V_{r} \geq 17$ leading to a primarily 1-DOF in-line response with a nearly constant $A_{x} / D$ (Fig. 3a). For higher KC, both $A_{y} / D$ and $A_{x} / D$ come into play across the considered $V_{r}$ range. These trends, which are quite sensitive to the $n$ change, are also captured by the wake oscillators. Nevertheless, considerable quantitative errors can be noticed for $n=3-4$ in Fig. 3a (KC=10), $n=5-6$ in Fig. $3 \mathrm{~b}(\mathrm{KC}=20)$ and all $n$ in Fig. $3 \mathrm{c}(\mathrm{KC}=40)$. Since we use the maximum $A_{y} / D$ and overall 2-DOF VIV features (averaged $A_{x} / D$ and $A_{y} / D$ trends) across $1<V_{r}<30$ as the governing calibration criteria, the most suitable qualitative and quantitative comparisons between wake oscillator and CFD models occur when $n=2,4$ and 8 for $\mathrm{KC}=10$, 20 and 40, respectively, in agreement with what is suggested by Sarpkaya (1976) for such a low Re range.

Figure 4 illustrates the effect of cylinder stiffness nonlinearities (i.e. linear vs. nonlinear oscillators) as well as the effect of wake-cylinder (acceleration, velocity and displacement) coupling terms on numerical predictions in comparison with CFD results. By considering $x-y$ linear oscillators $\left(\alpha_{x}=\beta_{x}=\alpha_{y}=\beta_{y}=0\right)$ for $\mathrm{KC}=10$, the predicted 
$A_{x} / D$ peak in Fig. 4 a is slightly shifted to the left due to the omitted cubic-type hardening stiffness whereas the predicted $A_{y} / D$ response in Fig. 4b becomes relatively negligible when $V_{r}$ exceeds 10, which is a substantially lower value than 17 , which corresponds to the CFD approach. The multi-peak $A_{y} / D$ feature disappears with the linear model. Such qualitative and quantitative errors would become greater for higher KC. As for the cylinder coupling or feedback terms in Eqs. (12) and (14), the exemplified in-line response at $\mathrm{KC}=10$ in Fig. $4 \mathrm{c}$ appears to be almost identical when applying either the cylinder displacement coupling $\left(f_{c x}=\Lambda_{x} x, f_{c y}=\Lambda_{y} y\right)$, velocity coupling $\left(f_{c x}=\Lambda_{x} \dot{x}, f_{c y}=\Lambda_{y} \dot{y}\right)$ or acceleration coupling $\left(f_{c x}=\Lambda_{x} \ddot{x}, f_{c y}=\Lambda_{y} \ddot{y}\right)$ terms. This implies a negligible effect of the coupling type on $A_{x} / D$. On the other hand, $A_{y} / D$ responses are much more affected by the wake-cylinder coupling choice as displayed in Fig. 4d, e and $\mathrm{f}$ for $\mathrm{KC}=10,20$ and 40, respectively. In all $\mathrm{KC}$ cases and based on the proposed set of empirical coefficients, the displacement coupling terms entail the worst prediction with considerable errors when compared with CFD results. Indeed, such different effects in applying the coupling terms reflect the distinct natures of in-line vs. cross-flow responses, dependent on $\mathrm{KC}$ and $V_{r}$. For oscillatory flow, the cylinder coupling type in the in-line wake oscillator has been found to play a minor or even negligible role in affecting the feature of in-line response amplitudes because the in-line response is primarily governed by the oscillatory flow frequency. This is different from the associated cross-flow response which is primarily governed by the vortex-induced lift force and associated excitation frequency. Nevertheless, cross-flow and in-line responses are dynamically coupled through the nonlinear terms of hydrodynamic $(q \dot{y}, p \dot{x}, q \dot{x}, p \dot{y})$, geometric $\left(x y^{2}, y x^{2}\right)$ and relative velocities $\left(f_{h}, \lambda\right)$. By accounting for the same and consistent wake-cylinder coupling type in both drag and lift wake oscillators, the prediction results suggest how the acceleration coupling terms are most suitable for oscillatory flow VIV as in steady flow VIV cases (Facchinetti et al., 2004; Srinil et al., 2018).

By accounting for the stiffness nonlinearities and acceleration coupling terms, and assigning the fixed values $C_{a}=1$ and $C_{D 0}=0.2$, Figure 5 illustrates the effect of varying hydrodynamic coefficients governing the inertia $\left(C_{M}\right)$, Morison drag $\left(C_{D M}\right)$ and oscillatory lift $\left(C_{L 0}\right)$ forces on the wake oscillator prediction in comparison with CFD results. For $\mathrm{KC}=10, A_{x} / D$ (Fig. $5 \mathrm{a}$ ) and $A_{y} / D$ (Fig. $5 \mathrm{~b}$ ) responses appear to be qualitatively similar (i.e. with the bend-to-right $\left(A_{x} / D\right)$ and multi-peak $\left(A_{y} / D\right)$ features) but quantitatively different with $C_{M}=0.5,1$ and 2 . Since $C_{M}$ term only appears in Eq. (15) governing the in-line loading, the changes in both $x-y$ responses as $C_{M}$ is varied suggest a two-dimensional dynamic coupling captured by the model. As for $C_{D M}$ which governs the in-line loading in Eq. (15) as well as the $x-y$ hydrodynamic damping contribution in Eq. (17), the compared $A_{x} / D$ and $A_{y} / D$ responses are shown in Fig. $5 \mathrm{c}$ and d, respectively, for $\mathrm{KC}=40$. Results indicate a greater effect of varying $C_{D M}$ on $A_{x} / D$ than $A_{y} / D$. That is, $A_{x} / D$ tends to increase by a faster rate when increasing $C_{D M}$ from 1.5 to 2.5 and increasing $V_{r}$ from 5 to 30 whereas the maximum $A_{y} / D$ remains quantitatively comparable. Note that it was not feasible to capture the CFD-based parabolic $A_{y} / D$ response shape for $10<V_{r}<25$ in Fig. 5 d by using a single set of empirical coefficients when varying $V_{r}$. A second set of empirical coefficients may be introduced for this range. The effect of $C_{L 0}$ on $A_{x} / D$ (Fig. 5e) and $A_{y} / D$ (Fig. 5f) is next discussed for $\mathrm{KC}=40$, recalling that $C_{L 0}$ affects both $x-y$ responses through $M_{L}$ in Eqs. (15) and (16). According to Sarpkaya (2010), the maximum $C_{L 0}$ is in the range 
0.3-1.5 for $\mathrm{KC}=40$, depending on $\beta\left(\mathrm{Re}\right.$ ). By way of tuning examples, $C_{L 0}=0.3,1.5$ and $1.5 / \sqrt{2}$ (root-mean squared value) are employed and compared. It is seen that the $C_{L 0}$ variation has a significant quantitative effect on the predicted $x-y$ responses. Based on the present wake oscillator calibration, $C_{L 0}=0.3$ appears to be the most suitable tuning coefficient, in conjunction with the recommended $C_{M}=1, C_{D M}=2, C_{a}=1$ and $C_{D 0}=0.2$. These empirical values and those described in the above second paragraph (Eqs. 18 and 19) will be used. In the Appendix, application of the present 2-DOF wake oscillator model and calibrated empirical coefficients to predict 1-DOF cross-flow or in-line VIV responses is further demonstrated and discussed in comparison with experimental results. In the following, numerical predictions of 2-DOF VIV predictions in oscillatory flows are presented.

\section{Oscillatory Flow VIV Prediction Characteristics}

Parametric investigations to predict 2-DOF VIV responses in oscillatory flows are now carried out for a wide range of system parameters (KC, $V_{r}, m^{*}, f_{r}$ and Re). As the presented wake oscillators have not been derived from the Navier-Stokes equations describing actual fluid mechanics, the numerical predictions - which have been calibrated with specific CFD results - need to be further validated with new future experimental tests to justify the model validity and identify its limitations. Nevertheless, some prediction insights can be gained in regard to 2-DOF response amplitudes, time histories, oscillation frequencies and motion trajectories, which allow one to realize the feasibility of applying wake oscillators to predict oscillatory flow VIV. In the following, response frequencies and amplitudes based on several model solutions are presented and discussed.

\subsection{Multi-Frequency Responses}

With $m^{*}=1.62, \xi=0.012$ and $V_{r}=7.5$, Fig. 6 presents in-line and cross-flow time histories for $\mathrm{KC}=10(n=2), 20$ $(n=4)$ and $40(n=8)$ whereas Fig. 7 presents the associated phase plane (displacement-velocity) portraits and $x$ $y$ trajectories (about 16 cycles), see also Fig. 3 for their response amplitudes. At low $\mathrm{KC}=10\left(\mathrm{KC} / V_{r}=f_{n} / f_{w}=f_{r}=\right.$ 1.33), the in-line response in Fig. 6a appears to have a zero mean value and a single harmonic frequency associated with the flow excitation whereas the cross-flow response in Fig. $6 \mathrm{~b}$ signals the fluctuating maximum/minimum amplitude components as well as competing frequencies. The in-line response is indeed driven by $f_{w}$ through the Morison drag $\left(C_{D M}\right)$ and inertia $\left(C_{M}\right)$ forces in Eq. (15). This observation is in qualitative agreement with the study of Anagnostopoulos and Iliadis (1998). Accordingly, the $x$ phase plane plot in Fig. 7a appears to be a nearly circular closed orbit. In contrast, the cross-flow response is governed by multiple nonlinear/linear forcing terms and frequencies (i.e. StKC $=f_{v} / f_{w}=2$ vs. $f_{n} / f_{w}=1.33$ ) involving wake oscillators in Eq. (16). This leads to the $y$ multiple orbital phase plane in Fig. 7b. By denoting $f_{x}$ and $f_{y}$ as the dimensional $x$ and $y$ oscillation frequencies, $f_{x} / f_{w}=N_{x}=$ 1 in Fig. 6a whereas the dominant $f_{y} / f_{w}=1.43$ in Fig. 6b suggesting a quasi-periodic response. Hence, the noninteger $x-y$ frequency ratio entails irregular or non-repeatable $x-y$ trajectories in Fig. 7c. As KC is increased to 20 $\left(f_{r}=2.67\right)$, the in-line response in Fig. $6 \mathrm{c}$ begins to be influenced by the wake oscillator frequency $(\mathrm{StKC}=4)$ leading to the distorted amplitudes and non-zero mean values. Therefore, the associated $x$ phase plane orbit is modulated, non-circular and asymmetric in Fig. 7d. Highly modulated $y$ responses with greater broadband frequency contents are expected as shown in Figs. $6 \mathrm{~d}$ and 7e, due to the enhanced nonlinearities. By further 
increasing $\mathrm{KC}$ to $40\left(\mathrm{KC} / V_{r}=f_{r}=5.33\right)$, the highly modulated $x$ and intermittent $y$ responses are clearly visible in Figs. 6e, 6f, $7 \mathrm{~g}$ and 7h, suggesting a chaotic-like VIV. Accordingly, $x-y$ trajectories become highly irregular in Fig. $7 \mathrm{i}$ exhibiting the symmetric $y$ vs. asymmetric $x$ (with a downstream drift) trajectories response with respect to the initial equilibrium at $x=y=0$. These features have also been experimentally observed by Lipsett and Williamson (1994) for high $f_{r}>4$ values.

Figure 8 presents the normalized $x-y$ response frequencies $\left(f_{x} / f_{w}, f_{y} / f_{w}\right)$ of the oscillating cylinder subject to oscillatory flows based on the Fast Fourier Transform (FFT) analysis, in association with the amplitudes in Fig. 3. Herein, a primary or dominant oscillation frequency corresponds to a highest peak with a normalized spectral amplitude of unity in FFT plots. In all $\mathrm{KC}=10,20$ and 40 cases, the dominant $f_{x} / f_{w}$ values in Fig. $8 \mathrm{a}, \mathrm{c}$ and e appear to be equal to unity (i.e. $N_{x}=1$, see also Eq. 3 ) as $V_{r}$ is increased. This is expected, agreeing with CFD results, indicating the governing sinusoidal flow excitation for $x$ responses. Owing to the coupling of oscillatory lift-drag forces, there are several in-line response frequency peaks with variable amplitudes of the power spectral density as illustrated in Fig. $6 \mathrm{a}(\mathrm{KC}=10), \mathrm{c}(\mathrm{KC}=20)$ and e $(\mathrm{KC}=40)$. The $f_{x} / f_{w}$ components of the first three peaks, ranked by their spectral density amplitudes, are summarized in Fig. 9a, c and e. For cross-flow response, $f_{y} / f_{w}$ patterns and amplitudes vary substantially with $V_{r}$ as well as KC. This reflects the dependence on both $V_{r}$ and $\mathrm{KC}$ as suggested by Zhao (2013), Bearman and Mackwood (1991). At $\mathrm{KC}=10, f_{y} / f_{w}$ values decrease monotonically as $V_{r}$ is increased until $A_{y} / D$ becomes trivial (Fig. 3b) as $V_{r}>15$ in Fig. 8b. Since the predicted cross-flow responses become highly modulated when increasing KC (Figs. 6 and 7), $f_{y} / f_{w}$ plots for $\mathrm{KC}=20$ (Fig. 8d) and 40 (Fig. 8f) reveal several peaks with variable amplitudes. This echoes the effect of system coupling and nonlinearities through the higher-order term $f_{h}$ in Eqs. (15) and (16) governing the flow-cylinder relative velocities. In all $\mathrm{KC}$ cases, $f_{y} / f_{w}$ values appear to be mostly non-integers. Figures $9 \mathrm{~b}, \mathrm{~d}$ and $\mathrm{f}$ show the variation of the dominant $f_{y} / f_{w}$ versus $V_{r}$ for all $\mathrm{KC}$, in comparison with CFD results (Zhao, 2013). Qualitatively speaking, the predicted $f_{y} / f_{w}$ values appear to increase (decrease) with $\mathrm{KC}\left(V_{r}\right)$ as in the CFD study. However, discrepancies between the two approaches are noticed, increasing with KC. A satisfactory agreement may be justified in the limited range of $13<V_{r}<20$ for KC $=10,7<V_{r}<13$ and $20<V_{r}<26$ for $\mathrm{KC}=20$. The present wake oscillator model is unable to capture actual integer $N_{y}$ values of $f_{y} / f_{w}$, leading to non-repeatable $x-y$ trajectories as shown in Fig. 7. In other words, $N_{y}$ is not necessarily equal to the specified $n$ for each $\mathrm{KC}$, suggesting how the oscillation frequencies are nonlinearly amplitude-dependent.

By computing $f_{y} / f_{n}=N_{y}\left(V_{r} / \mathrm{KC}\right)$ as in Eq. (3) based on the obtained primary $f_{y} / f_{w}$ or $N_{y}$ which may be integer or non-integer (Fig. 9), Figure 10 plots $f_{y} / f_{n}$ versus $V_{r}$ in comparison with CFD results and the reference lines with a perfect integer $N_{y}$. It is also worth referring to the experimental study of cross-flow only VIV by Sumer and Fredsoe (1988) who reported that, for $\mathrm{KC}=10, N_{y}=2$ throughout the $V_{r}$ range; for $\mathrm{KC}=20$, the primary $f_{y} / f_{n}$ follows the lines of $N_{y}=4,3$ and 2 in the approximated range of $2<V_{r}<6,6.5<V_{r}<8.5$ and $8.5<V_{r}<15$, respectively. For the 2-DOF VIV study of Zhao (2013), the primary $f_{y} / f_{n}$ for $\mathrm{KC}=10$ follows the lines of $N_{y}=2\left(V_{r}<14\right)$ and $1\left(V_{r} \geq 14\right)$; the primary $f_{y} / f_{n}$ for $\mathrm{KC}=20$ follows the lines of $N_{y}=4,3$ and 1 and then jumps up to $N_{y}=2$ in the 
approximate range $4<V_{r}<7,8<V_{r}<13,14<V_{r}<19$ and $20<V_{r}<30$, respectively, as in Fig. $10 \mathrm{~b}$. Notwithstanding a slight difference of $m^{*} \xi$, experimental (1-DOF) and numerical CFD (2-DOF) results do not perfectly share the same cross-flow response characteristics. Results from the wake oscillator model capture a gradual decreasing $f_{y} / f_{n}$ shift - instead of a rapid downward or upward $f_{y} / f_{n}$ jump in CFD plots - along $N$ lines as $V_{r}$ is increased. Both CFD and wake oscillator results in Fig. 10a and b reveal $N_{y}=1$ for $\mathrm{KC}=10$ and 20 at some $V_{r}$ ranges, implying that the cross-flow response is governed by the inline oscillatory excitation frequency $f_{w}$.

\subsection{Bi-Parametric Response Contours}

In previous Sections 3 and 4.1, the predicted 2-DOF VIV responses in oscillatory flows in the case of varying $V_{r}$ ( $U_{m}$ and $f_{w}$ ) are based on a fixed $\mathrm{KC}(n), m^{*}$ and $\xi$, with the purpose of empirical tuning and comparison of response amplitudes and frequencies with CFD results (Zhao, 2013). Here, the effects of system fluid-structure parameters in wider ranges are further parametrically explored in order to better understand the prediction capability of the present wake oscillators and chosen empirical coefficients.

Figure 11 presents contour plots of $A_{x} / D$ and $A_{y} / D$ as functions of both $m^{*}$ [0.1-10] and $V_{r}$ [0.1-30] for $\mathrm{KC}=10(n=2), 20(n=4)$ and $40(n=8)$, while $\xi=0.012$ as in Fig. 3 whose $m^{*}=1.62$. For $\mathrm{KC}=10, A_{x} / D$ peaks in Fig. 11a appear to decrease and slightly shift to a lower $V_{r}$ as $m^{*}$ is increased, maintaining a linear resonancelike feature around $10<V_{r}<15$. The multi-peak feature of the associated $A_{y} / D$ response in Fig. $11 \mathrm{~b}$ is greatly affected by the $m^{*}$ change, disappearing as $m^{*} \approx 6$ leading to a considerably reduced range of appreciable $A_{y} / D$ centered around $V_{r} \approx 10$. For $\mathrm{KC}=20$, there is a range of $m^{*}>6$ in which $A_{x} / D$ peaks take place within the considered $V_{r}$ range as shown in Fig. 11c. The associated $A_{y} / D$ response in Fig. $11 \mathrm{~d}$ reveals two distinct $m^{*}-V_{r}$ boundaries separated around $V_{r} \approx 10$, in which comparable peak $A_{y} / D$ values are found around $V_{r} \approx 7$ and 15 . Again, such a multi-peak feature disappears as $m^{*}$ is increased to about 8 being greater than 6 in the lower $\mathrm{KC}=10$ case in Fig. 11a. For $\mathrm{KC}=40$, overall $A_{x} / D$ responses in Fig. 11e increase with $V_{r}$ for all $m^{*}$, without showing a peak in the considered $V_{r}$ range. For $A_{y} / D$ responses, the two $m^{*}-V_{r}$ boundaries, shown in Fig. $11 \mathrm{~d}$ for $\mathrm{KC}=20$, tend to merge and result in a plateau amplitude response in a large $V_{r}$ range in Fig. 11f. This is due to the amplified responses as $\mathrm{KC}$ is increased in the low $m^{*}<6$ range. However, the $A_{y} / D$ response becomes limited in a small $V_{r}$ range as $m^{*}>8$, for which its peak is centered around $V_{r}=5$ resembling that occurring in a steady flow VIV. This implies that, for higher $m^{*}$ and higher $\mathrm{KC}$ cases where the drag is increasingly dominated, $A_{y} / D$ response behaves similarly to VIV in steady flow. For the considered $m^{*}$ range, the predicted maximum $A_{y} / D$ does not necessarily correspond to the lowest $m^{*}$ case for higher $\mathrm{KC}=20$ and 40. This suggests a bi-parametric $m^{*}$-KC dependence. Overall, the maximum $A_{x} / D \approx 2.5,3.5$ and 6 times the maximum $A_{y} / D(\leq 1)$ for $\mathrm{KC}=10,20$ and 40 , respectively.

In contrast to Fig. 11 where $\mathrm{KC}$ as well as $n$ is fixed while varying $V_{r}$, Figure 12 presents contour plots of $A_{x} / D$ and $A_{y} / D$ as functions of both KC [10-60] and $V_{r}[0.1-30]$ for a given $m^{*}=1.62$ and 4 while $\xi=0.012 . U_{m}$ is the only parameter being varied in this scenario. Following Sarpkaya (1976), it may be assumed that $n=2,3,4$, $5,6,7,8,9,10$ and 11 for $10 \leq \mathrm{KC}<15,15 \leq \mathrm{KC}<20,20 \leq \mathrm{KC}<25,25 \leq \mathrm{KC}<30,30 \leq \mathrm{KC}<35,35 \leq \mathrm{KC}<$ 
$40,40 \leq \mathrm{KC}<45,45 \leq \mathrm{KC}<50,50 \leq \mathrm{KC}<55$ and $55 \leq \mathrm{KC}<60$, respectively. For lower $m^{*}=1.62$, the $A_{x} / D$ response in Fig. 12a increases as $V_{r}$ is increased for a given KC. However, $A_{x} / D$ peaks appear to be more sensitive to the $\mathrm{KC}$ as well as $n$ change when $V_{r}>15$ than when $V_{r}<15$. For higher $m^{*}=4, A_{x} / D$ peaks in Fig. $12 \mathrm{c}$ appear to be largely independent of $\mathrm{KC}$ for a given $V_{r}$. These features are distinct from the associated $A_{y} / D$ responses in Fig. $12 \mathrm{~b}\left(m^{*}=1.62\right)$ and $12 \mathrm{~d}\left(m^{*}=4\right)$ where variations in both $V_{r}$ and $\mathrm{KC}$ affect $A_{y} / D$ peaks in a stepwise fashion because of varying $n$. The lower $m^{*}$ case produces the overall greater $A_{y} / D$ responses as expected. As in Fig. 11, results in Fig. $12 \mathrm{~d}$ suggest that $A_{y} / D$ peaks occur around $V_{r} \approx 5$ as $\mathrm{KC}(n)$ and $m^{*}$ are both increased. Although the variable $n$ affects the wake oscillators in Eqs. (12) and (14), the maximum $A_{y} / D$ is still limited to be less than 1.2.

Next, instead of directly specifying an integer $n$ (as $n=\mathrm{StKC}$ ) regardless of actual St, a parametric study may be carried out by assigning and keeping St fixed. In so doing, we specify St $=0.2$ (Sumer and Fredsoe, 2006). Since previous experimental (Lipsett and Williamson, 1994) and CFD (Zhao, 2013) studies highlighted the oscillatory flow VIV dependence on both $\mathrm{KC}$ and $V_{r}$, one may assign and vary the frequency ratio $f_{r}=f_{n} / f_{w}=\mathrm{KC} / V_{r}$ (Eq. 2) and the associated KC through StKC in Eqs. (12) and (14). The aim is to determine if a nonlinear resonance between the two sets of wake-cylinder nonlinear oscillators might be achieved. By specifying the ranges of $1<f_{r}<4$ and $0.5<V_{r}<30$, the associated $\mathrm{KC}$ variation $\left(\mathrm{KC}=f_{r} V_{r}\right)$ from 0.75 to 120 is shown in Fig. 13 whereas contour plots of the predicted $A_{x} / D$ and $A_{y} / D$ are displayed in Fig. 14 for $m^{*}=1.62(\mathrm{a}, \mathrm{b})$ and $4(\mathrm{c}, \mathrm{d})$. It is seen that $A_{x} / D$ responses in both Fig. $14 \mathrm{a}$ and $14 \mathrm{c}$ increase as both $V_{r}$ and $\mathrm{KC}$ are increased for a given $f_{r}$, the latter having a greater effect on the system with lower $m^{*}=1.6$. For $m^{*}=4, A_{x} / D$ response appears to be relatively independent of varying $f_{r}$ : this feature is similar to the varying $n$ case in Fig. $12 \mathrm{c}$ vs. $12 \mathrm{a}$. On the other hand, $A_{y} / D$ responses in both Fig. $14 \mathrm{~b}$ and $14 \mathrm{~d}$ appear to be strongly dependent on $f_{r}, V_{r}, \mathrm{KC}$ and $m^{*}$; their features are qualitatively and quantitatively different from those in Fig. 12b and 12d. The $f_{r}-V_{r}$ boundary for the maximum $A_{y} / D$ peaks is found to be greater and wider in the case of lower $m^{*}$ as shown in Fig. 14b. In association with Fig. 14, a table in Fig. 13 summarizes $A_{y} / D$ peak values, associated $V_{r}$ and $\mathrm{KC}$ for each specified $f_{r}$ and $m^{*}$. It is seen that the averaged maximum $A_{y} / D \approx 1$ and 0.68 occurs in the range of $16<V_{r}<21$ and $6<V_{r}<10$ for $m^{*}=1.62$ and 4, respectively, suggesting a strong influence of $m^{*}$ in affecting $A_{y} / D$ peaks and shifting the critical $V_{r}$ range, consistently with results in Fig. 11. The corresponding $\mathrm{KC}$ increases such that $\mathrm{KC} / V_{r} \approx f_{r}$.

Finally, the combined effects of Re and $\mathrm{KC}$ on the prediction of 2-DOF VIV responses in oscillatory flows are studied. For a specific $\beta=\operatorname{Re} / \mathrm{KC}=1107$, the hydrodynamic $\operatorname{drag}\left(C_{D M}\right), \operatorname{lift}\left(C_{L 0}\right)$ and inertia $\left(C_{M}\right)$ coefficients are plotted versus KC, as an example, in Fig. 15a based on data of Sarpkaya (1976). It is seen that $C_{D M}$ and $C_{L 0}$ $\left(C_{M}\right)$ increase (decreases) with increasing $\mathrm{KC}$ as well as $\mathrm{Re}$ for the inertia-dominated system $(\mathrm{KC}<10)$. After reaching a peak, both $C_{D M}$ and $C_{L 0}$ decrease when further increasing $\mathrm{KC}$ towards the drag-dominated region (KC > 10). In contrast, the associated $C_{M}$ increases until approaching a limiting value of about 1.5. Such inertia-drag transitional behaviour is also applicable to other lower and higher $\beta$ (Sarpkaya, 1976). To demonstrate the dependence on $\mathrm{KC}$ and $\operatorname{Re}$ (through a fixed $\beta=1107$ ), the following exponential (exp) fitting functions associated with Fig. 15a for $6<\mathrm{KC}<80$ may be introduced and incorporated into the simulation model. 


$$
\begin{aligned}
& C_{D M}=0.43 \exp \left[-((\mathrm{KC}-13.44) / 6.47)^{2}\right]+1734 \exp \left[-((\mathrm{KC}+3907) / 1460)^{2}\right]+0.34 \exp \left[-((\mathrm{KC}-14.05) / 16.95)^{2}\right], \\
& C_{L 0}=1.39 \exp \left[-((\mathrm{KC}-11.64) / 6.48)^{2}\right]+1.04 \exp \left[-((\mathrm{KC}-17.44) / 14.69)^{2}\right]+7.04 \times 10^{13} \exp \left[-((\mathrm{KC}+3196) / 569.5)^{2}\right], \\
& C_{M}=1.40 \exp \left[-((\mathrm{KC}-5.65) / 3.25)^{2}\right]+0.71 \exp \left[-((\mathrm{KC}-30.39) / 27.60)^{2}\right]+1.40 \exp \left[-((\mathrm{KC}-87.28) / 59.65)^{2}\right] .
\end{aligned}
$$

For a given $1.5<f_{r}<4$ range, the associated $V_{r}$ can be specified and varied through $V_{r}=\mathrm{KC} / f_{r}$ for a given $6<\mathrm{KC}<80(5535<\mathrm{Re}<88560)$ range, as shown in Fig. $15 \mathrm{~b}$. The minimum and maximum $V_{r}$ is about 3.33 and 53.33 , respectively. By assigning $m^{*}=1.62, \xi=0.012$, accounting for the StKC variation in Eqs. (12) and (14) with $\mathrm{St}=0.2$, functions in above $\mathrm{Eq}$ (20) for a fixed $\beta=1107$, and keeping other empirical coefficients unchanged as before, the predicted response contours of $A_{x} / D$ and $A_{x} / D$ are presented in Fig. $15 \mathrm{c}$ and d, respectively. For in-line responses, the maximum $A_{x} / D$ increases with increasing $\mathrm{KC}(\mathrm{Re})$ for a given $f_{r}$ whereas it decreases with increasing $f_{r}$ for a given $\mathrm{KC}$ since $V_{r}$ is decreased. For cross-flow responses, the maximum $A_{y} / D$ values strongly depend on $f_{r}$ $\left(V_{r}\right)$ and $\mathrm{KC}(\mathrm{Re})$ whose contour plots display a complex modulated response map with multi-peak features when varying either $\mathrm{KC}$ or $f_{r}$. The absolute maximum $A_{y} / D$ (up to $\approx 2.5$ ) occurs in the intermediate $\mathrm{KC}$ and high $f_{r}$ (low $V_{r}$ ) regions. In comparison with results - which disregard the Re dependence of hydrodynamic coefficients in the prediction model - in Figs. 12 and 14 in which $308<\operatorname{Re}<9240$ and $0<V_{r}<30$, the maximum $A_{x} / D \approx 7$ in the range of $V_{r}<30$ occurs at $\mathrm{KC} \approx 40$ and $f_{r} \approx 1.5\left(V_{r} \approx 26.7\right)$ in Fig. $15 \mathrm{c}$, while the maximum $A_{y} / D \approx 2.5$ in the range of $V_{r}<30$ occurs at $\mathrm{KC} \approx 37$ and $f_{r} \approx 3.9\left(V_{r} \approx 9.5\right)$ in Fig. $15 \mathrm{~d}$. The associated greater $\mathrm{Re}=44280(\mathrm{KC}=40)$ and $40959(\mathrm{KC}=37)$ values entail greater $x-y$ responses in Fig. $15 \mathrm{c}$ and $\mathrm{d}$ when accounting for both $\mathrm{Re}$ and $\mathrm{KC}$ effects in the prediction model than those in Figs. 12a, 12b, 14a and 14b (for the same range of $10<\mathrm{KC}<60$ ) when accounting for only the $\mathrm{KC}$ effect in the prediction model. Such trend of increasing responses with increasing $\mathrm{Re}$ has been observed in steady flow VIV cases, see, e.g., Blevins and Coughran (2009). Nevertheless, there is a need to justify such oscillatory flow VIV predictions with associated experimental tests or CFD simulations which are currently scarce for a low $m^{*}$ system in a high Re flow regime.

\section{Conclusions}

A time-domain simulation model based on nonlinear wake-cylinder oscillators to predict combined cross-flow and in-line VIV of an elastically mounted circular cylinder in planar and oscillatory flow has been presented. This approach is interestingly new since wake oscillators have typically been applied to steady flow VIV predictions. Herein, the time-varying relative flow-cylinder velocities and accelerations have been accounted for in formulating the coupled hydrodynamic lift, drag and inertia forces with explicit time-dependent and hybrid trigonometric terms leading to two-dimensional cylinder oscillations excited by oscillatory flow. Depending on $\mathrm{KC}$ incorporating the flow maximum velocity and excitation frequency, a set of model empirical coefficients have been calibrated by tuning response amplitudes with the limited CFD simulation data in the literature for a low mass-damping system subject to specific $\mathrm{KC}=10,20$ and 40, $0<V_{r}<30$ and $\mathrm{Re} / V_{r}=308$. Dual wake oscillators with cylinder acceleration coupling terms are recommended. Empirical functions depending on $\mathrm{KC}$ and mass ratio have been introduced and applied to parametrically investigate the influence of system parameters on response prediction characteristics. 
Three feasible model solutions have been investigated in cases of varying $\mathrm{KC}$ and $V_{r}$, including (i) the assumed and fixed number of lift force oscillations per flow cycle $(n=\mathrm{StKC}=$ constant $)$ depending on the $\mathrm{KC}$ range, (ii) the assumed St and specified cylinder-to-flow frequency ratio $f_{r}\left(f_{r}=\mathrm{KC} / V_{r}\right)$ and $V_{r}$ range, and (iii) the specified Stokes parameter $\beta$ for given $\mathrm{KC}(\beta=\mathrm{Re} / \mathrm{KC})$ and $f_{r}$ ranges. In the latter case, hydrodynamic coefficients governing the drag, lift and inertia forces are varied as function of $\mathrm{KC}$ and Re based on experimental data of oscillatory flows past circular cylinders. These solution scenarios enable insights into a variety of predicted response boundaries with bi-parametric $\left(m^{*}-V_{r}, \mathrm{KC}-V_{r}, f_{r}-V_{r}, f_{r}\right.$-KC) contours of cross-flow and in-line amplitudes and their maximum/minimum values which may be useful for future comparisons and verifications by alternative numerical approaches and experiments. Overall, some qualitative features of oscillatory flow VIV have been captured by wake oscillators, including the increasing in-line response due primarily to the oscillatory flow frequency, the multi-peak cross-flow amplitude responses with fluctuating time histories due to multi-frequency components, the gradual shifts of cylinder oscillation-to-flow frequency ratios, and irregular motion trajectories due to the multi-directional flow velocities and multiple competing frequencies. The predicted dominant vibration frequencies in the in-line direction follow the sinusoidal flow excitation whereas those in the cross-flow direction are strongly dependent on $\mathrm{KC}, V_{r}$, system frequency ratios, mass ratio and nonlinearities. A main limitation of the present wake oscillators lies in the inability to capture the repetitive $x-y$ trajectories experimentally observed in some $\mathrm{KC}-V_{r}$ cases. This may call for a further development of three-dimensional and higher-order wake oscillators accounting for the cylinder spanwise dependence and the orbital flow motion effect.

\section{Declaration of competing interest}

The authors declare that they have no known competing financial interests or personal relationships that could have appeared to influence the work reported in this paper.

\section{CRediT authorship contribution statement}

Pierre-Adrien Opinel: Conceptualization, Methodology, Software, Validation, Formal analysis, Investigation, Writing - original draft, Visualization. Narakorn Srinil: Conceptualization, Methodology, Resources, Formal analysis, Writing - original draft, review \& editing, Supervision.

\section{Appendix: Prediction of 1-DOF VIV response in oscillatory flow}

Based on the present model in Section 2 and the calibrated empirical coefficients in Section 3, the system of Eqs. (11) and (12) or the system of Eqs. (13) and (14) is now applied to predict a 1-DOF in-line or cross-flow VIV response, respectively, in comparison with some experimental results in the literature as shown in Fig. 16.

For in-line response, experimental data of Williamson (1985) are considered for which $m^{*}=6.5, \xi=0.02$ and $V_{r} / \mathrm{KC}=0.87$, and those of Bearman and Mackwood (1991) are also considered for which $m^{*}=4.7,0.0007 \leq \xi \leq$ 0.06 (this range was experimentally given), and $V_{r} / \mathrm{KC}=1 / 1.83 \approx 0.546$. The tuned values of $C_{M}=1, C_{D M}=2, C_{a}$ $=1, C_{D 0}=0.2, \beta_{x}=0.7, \varepsilon_{x}=0.3, \Lambda_{x}=12$ and $\alpha_{x}$ in Eq. (18) are employed in the simulation model. In the case of varying $\mathrm{KC}$, we assign $n=2$ for $\mathrm{KC} \leq 15, n=3$ for $\mathrm{KC} \leq 20, n=4$ for $\mathrm{KC} \leq 25, n=5$ for $\mathrm{KC} \leq 30, n=6$ for $\mathrm{KC} \leq$ 
35, $n=7$ for $\mathrm{KC} \leq 40, n=8$ for $\mathrm{KC} \leq 45, n=9$ for $\mathrm{KC} \leq 50, n=10$ for $\mathrm{KC} \leq 55$, and $n=11$ for higher KC. Numerical and experimental results are compared in Fig. 16a for the maximum $x$ amplitudes $\left(A_{x} / D\right)$ and 16b for the root-mean-squared $x$ amplitudes $\left(A_{x, \mathrm{rms}} / D\right)$, exhibiting a good agreement in terms of the response values and the monotonically increasing trend as $\mathrm{KC}$ is varied. The $\xi$ value is seen to have a negligible effect on the predicted response in Fig. 16b. Hence, the set of in-line wake-structure oscillator coefficients from the 2-DOF VIV calibration may be applicable to the 1-DOF in-line VIV prediction.

For cross-flow response, experimental results of Sumer and Fredsoe (1988) are considered for which $m^{*}=$ $1.8, \xi=0.043$ and $\mathrm{KC}=20$. The tuned values of $C_{M}=1, C_{D M}=2, C_{a}=1, C_{L 0}=0.3, \alpha_{y}=0.7, \varepsilon_{y}=0.00234 \mathrm{e}^{0.228 \mathrm{~m}^{*}}$ and $\beta_{y}$ in Eq. (19), together with $n=4$, are employed in the simulation model. By considering $\Lambda_{y}=38$ deduced from the 2-DOF VIV calibration, Fig. 16c shows large discrepancies between numerical and experimental results in 1-DOF cross-flow amplitudes $\left(A_{y} / D\right)$ when varying $V_{r}$ although the multi-peak feature is noticeable. Hence, the set of cross-flow wake-structure oscillator coefficients from the 2-DOF VIV calibration may not be directly applicable to the 1-DOF cross-flow VIV prediction. A further attempt has been carried out by tuning $\Lambda_{y}$ governing the lift force oscillator to match the experimentally observed maximum responses and associated peaks at $V_{r} \approx 5.33$, 7.92, 11.85 and 12.36. Accordingly, by keeping other empirical values unchanged, it is found that $\Lambda_{y}=2$ for $V_{r} \leq$ $5, \Lambda_{y}=18$ for $5<V_{r} \leq 5.5, \Lambda_{y}=2$ for $5.5<V_{r} \leq 7, \Lambda_{y}=32$ for $7<V_{r} \leq 8.5, \Lambda_{y}=16$ for $8.5<V_{r} \leq 11, \Lambda_{y}=34$ for $11<V_{r} \leq 13$ and $\Lambda_{y}=20$ for $13<V_{r} \leq 16$, leading to an improved prediction as shown in Fig. 16d. This variation in empirical $\Lambda_{y}$ values reflects a difficulty in tuning only one empirical variable (as others are fixed) and in applying a unique set of empirical variables to predict VIV in the whole considered $V_{r}$ range. This is known as the key limiting capability of the wake oscillator. To overcome this challenge, a machine learning algorithm together with new experimental data and controlled parameters $\left(m^{*}, \xi, \mathrm{KC}, V_{r}, \mathrm{Re}\right)$ may be implemented. This is subject to our future research.

\section{References}

Anagnostopoulos, P., Iliadis, G., 1998. Numerical study of the flow pattern and the in-line response of a flexible cylinder in an oscillating stream. Journal of Fluids and Structures 12, 225-258.

Bai, X., Qin, W., 2014. Using vortex strength wake oscillator in modelling of vortex induced vibrations in two degrees of freedom. European Journal of Mechanics - B/Fluids 48, 165-173.

Bearman, P.W., Graham, J.M.R., Obasaju, E.D., 1984. A model equation for the transverse forces on cylinders in oscillatory flows. Applied Ocean Research 6, 166-172.

Bearman, P.W., Mackwood, P.R., 1991. Non-linear vibration characteristics of a cylinder in an oscillating water flow, The 5th Conference on Flow-Induced Vibrations, pp. 21-31.

Blevins, R.D., 1990. Flow-Induced Vibration. Van Nostrand Reinhold.

Blevins, R.D., Coughran, C.S., 2009. Experimental investigation of vortex-induced vibration in one and two dimensions with variable mass, damping, and Reynolds number. Journal of Fluids Engineering 131.

Facchinetti, M.L., De Langre, E., Biolley, F., 2004. Coupling of structure and wake oscillators in vortex-induced 
vibrations. Journal of Fluids and structures 19, 123-140.

Fu, B., Zou, L., Wan, D., 2018. Numerical study of vortex-induced vibrations of a flexible cylinder in an oscillatory flow. Journal of Fluids and Structures 77, 170-181.

Fu, S., Wang, J., Baarholm, R., Wu, J., Larsen, C.M., 2014. Features of vortex-induced vibration in oscillatory flow. Journal of Offshore Mechanics and Arctic Engineering 136, 011801.

Gao, Y., Zou, L., Zong, Z., Takagi, S., Kang, Y., 2019. Numerical prediction of vortex-induced vibrations of a long flexible cylinder in uniform and linear shear flows using a wake oscillator model. Ocean Engineering 171, 157-171.

Hartlen, R.T., Currie, I.G., 1970. Lift-oscillator model of vortex-induced vibration. Journal of the Engineering Mechanics Division 96, 577-591.

Hayashi, K., 1984. The Non-Linear Vortex-Excited Vibration of A Vertical Cylinder In Waves. University of Liverpool.

Justesen, P., 1991. A numerical study of oscillating flow around a circular cylinder. Journal of Fluid Mechanics 222, 157-196.

Kozakiewicz, A., Sumer, B.M., Fredsøe, J., Hansen, E.A., 1997. Vortex regimes around a freely vibrating cylinder in oscillatory flow. International Journal of Offshore and Polar Engineering 7, 94-103.

Lipsett, A.W., Williamson, I.D., 1994. Response of a cylinder in oscillatory flow. Journal of Fluids and Structures 8, 681-709.

Low, Y.M., Srinil, N., 2016. VIV fatigue reliability analysis of marine risers with uncertainties in the wake oscillator model. Engineering Structures 106, 96-108.

Morison, J.R., Johnson, J.W., Schaaf, S.A., 1950. The force exerted by surface waves on piles. Journal of Petroleum Technology 2, 149-154.

Obasaju, E.D., Bearman, P.W., Graham, J.M.R., 1988. A study of forces, circulation and vortex patterns around a circular cylinder in oscillating flow. Journal of Fluid Mechanics 196, 467-494.

Sarpkaya, T., 1976. Vortex shedding and resistance in harmonic flow about smooth and rough circular at high Reynolds numbers. Naval Postgraduate School, Monterey CA.

Sarpkaya, T., 1979. Vortex-induced oscillations: a selective review. Journal of Applied Mechanics 46, 241-258.

Sarpkaya, T., 2010. Wave Forces on Offshore Structures. Cambridge University Press.

Sarpkaya, T., Isaacson, M., 1981. Mechanics of Wave Forces on Offshore Structures. Van Nostrand Reinhold Company.

Srinil, N., 2010. Multi-mode interactions in vortex-induced vibrations of flexible curved/straight structures with geometric nonlinearities. Journal of Fluids and Structures 26, 1098-1122.

Srinil, N., 2011. Analysis and prediction of vortex-induced vibrations of variable-tension vertical risers in linearly sheared currents. Applied Ocean Research 33, 41-53.

Srinil, N., Opinel, P.-A., Tagliaferri, F., 2018. Empirical sensitivity of two-dimensional nonlinear wake-cylinder 
oscillators in cross-flow/in-line vortex-induced vibrations. Journal of Fluids and Structures 83, 310-338.

Srinil, N., Wiercigroch, M., O’Brien, P., 2009. Reduced-order modelling of vortex-induced vibration of catenary riser. Ocean Engineering 36, 1404-1414.

Srinil, N., Zanganeh, H., 2012. Modelling of coupled cross-flow/in-line vortex-induced vibrations using double Duffing and van der Pol oscillators. Ocean Engineering 53, 83-97.

Sumer, B.M., Fredsoe, J., 1988. Transverse vibrations of an elastically mounted cylinder exposed to an oscillating flow. Journal of Offshore Mechanics and Arctic Engineering 110, 387-394.

Sumer, B.M., Fredsoe, J., 1989. Effect of Reynolds number on vibrations of cylinders. Journal of Offshore Mechanics and Arctic Engineering 111, 131-137.

Sumer, B.M., Fredsoe, J., 2006. Hydrodynamics Around Cylindrical Strucures. World scientific.

Thorsen, M.J., Sævik, S., Larsen, C.M., 2016. Time domain simulation of vortex-induced vibrations in stationary and oscillating flows. Journal of Fluids and Structures 61, 1-19.

Ulveseter, J.V., Thorsen, M.J., Sævik, S., Larsen, C.M., 2018. Time domain simulation of riser VIV in current and irregular waves. Marine Structures 60, 241-260.

Wang, J., Fu, S., Baarholm, R., 2018. Evaluation of vortex-induced vibration of a steel catenary riser in steady current and vessel motion-induced oscillatory current. Journal of Fluids and Structures 82, 412-431.

Williamson, C.H.K., 1985. In-line response of a cylinder in oscillatory flow. Applied Ocean Research 7, 97-106.

Williamson, C.H.K., Govardhan, R., 2004. Vortex-induced vibrations. Annual Review of Fluid Mechanics 36, 413-455.

Williamson, I.D., 1991. Response of Cylinders to Oscillatory Flow, Department of Mechanical Engineering. University of Alberta, p. 108.

Xu, W.-H., Zeng, X.-H., Wu, Y.-X., 2008. High aspect ratio (L/D) riser VIV prediction using wake oscillator model. Ocean Engineering 35, 1769-1774.

Yang, W., Ai, Z., Zhang, X., Chang, X., Gou, R., 2018. Nonlinear dynamics of three-dimensional vortex-induced vibration prediction model for a flexible fluid-conveying pipe. International Journal of Mechanical Sciences 138139, 99-109.

Zanganeh, H., Srinil, N., 2014. Characterization of variable hydrodynamic coefficients and maximum responses in two-dimensional vortex-induced vibrations with dual resonances. Journal of Vibration and Acoustics 136, 051010 .

Zanganeh, H., Srinil, N., 2016. Three-dimensional VIV prediction model for a long flexible cylinder with axial dynamics and mean drag magnifications. Journal of Fluids and Structures 66, 127-146.

Zhao, M., 2013. Numerical investigation of two-degree-of-freedom vortex-induced vibration of a circular cylinder in oscillatory flow. Journal of Fluids and Structures 39, 41-59. 


\section{Highlights}

- Nonlinear wake oscillators are applied to two-degree-of-freedom VIV in oscillatory flows.

- Empirical coefficients and functions are proposed through calibration and sensitivity studies.

- Effects of Keulegan-Carpenter, reduced velocity, frequency and mass ratios are investigated.

- Feasible numerical solutions and associated bi-parametric response contours are presented.

- Model dependence of hydrodynamic coefficients on the Reynolds number is discussed. 


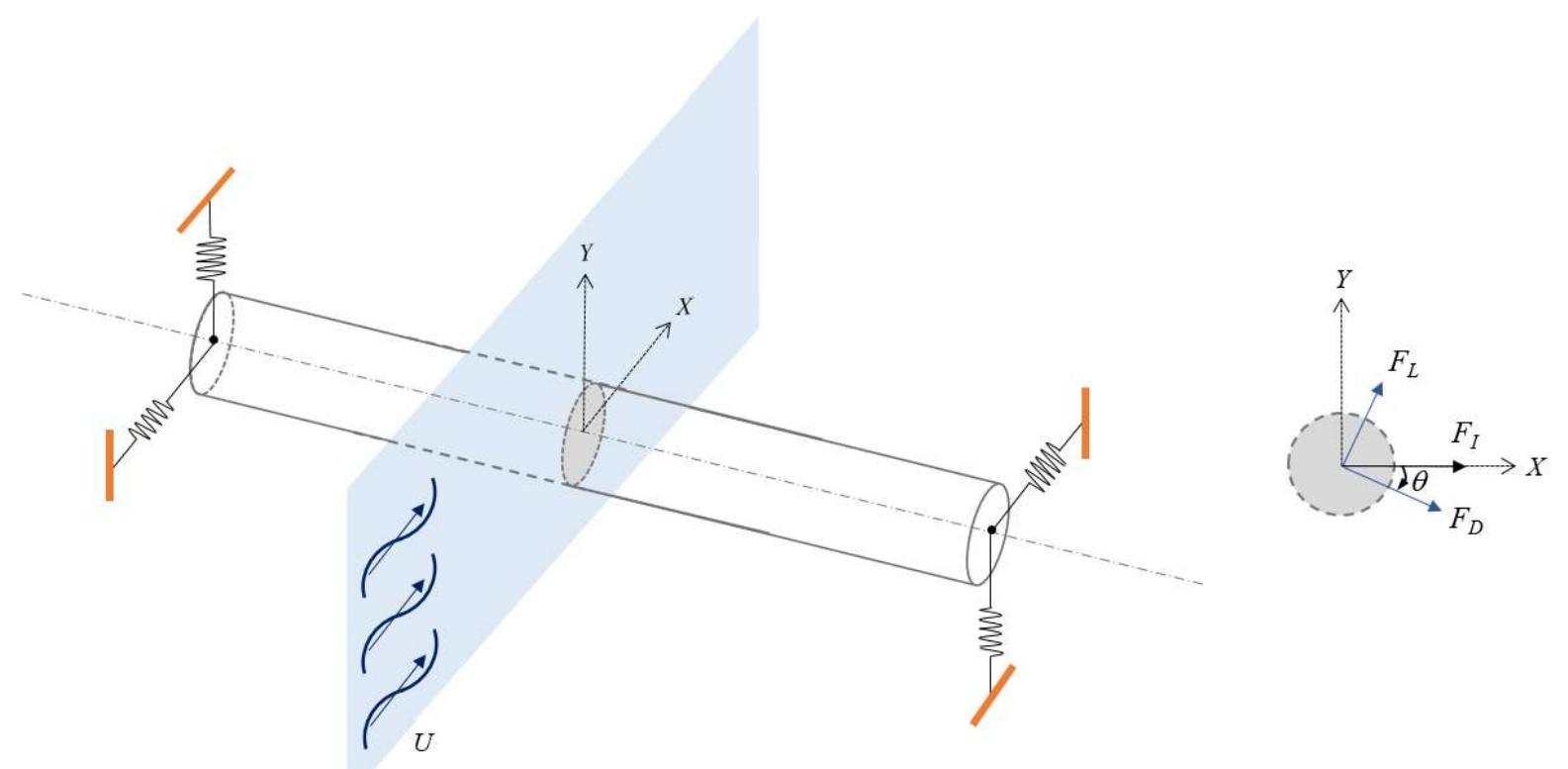

Figure 1. Schematic view of an elastically mounted circular cylinder in oscillatory flow, instantaneous direction $(\theta)$ of relative flow-cylinder velocities and hydrodynamic force components. 
(a)

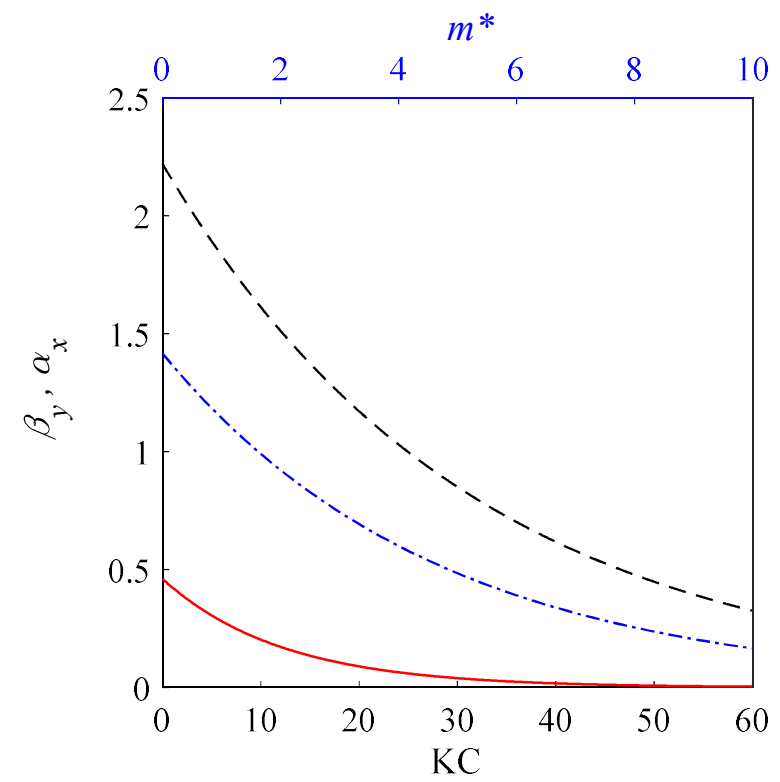

(b)

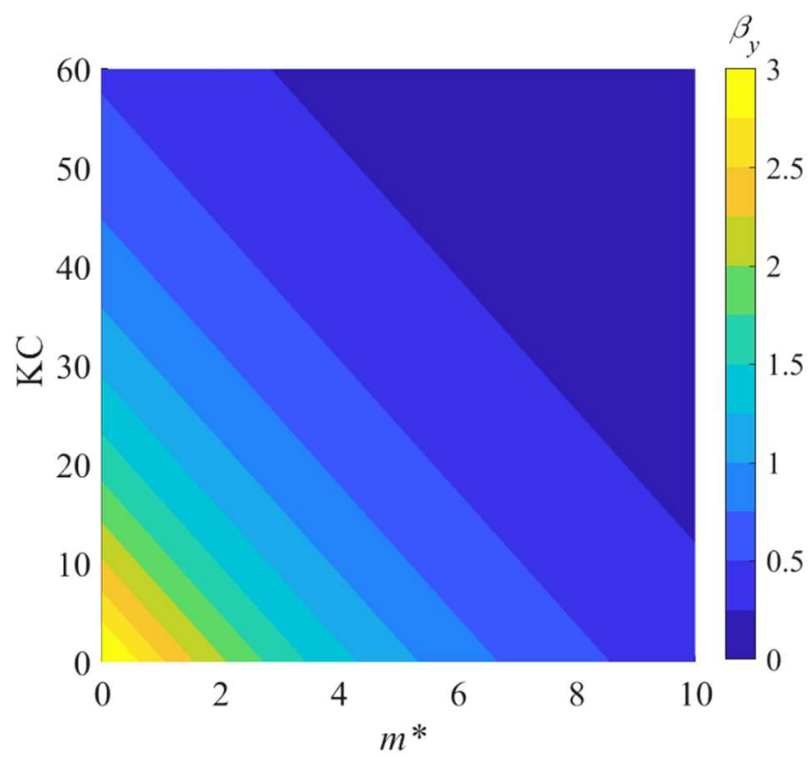

Figure 2. Empirical functions: (a) $\alpha_{x}$ (solid line) and $\beta_{y}$ (dashed line) dependent on $\mathrm{KC}$ for a given $m^{*}=1.62$, and $\beta_{y}$ (dotted-dashed line) dependent on $m^{*}$ for a given $\mathrm{KC}=10$; (b) $\beta_{y}$ dependent on both $\mathrm{KC}$ and $m^{*}$. 
(a)

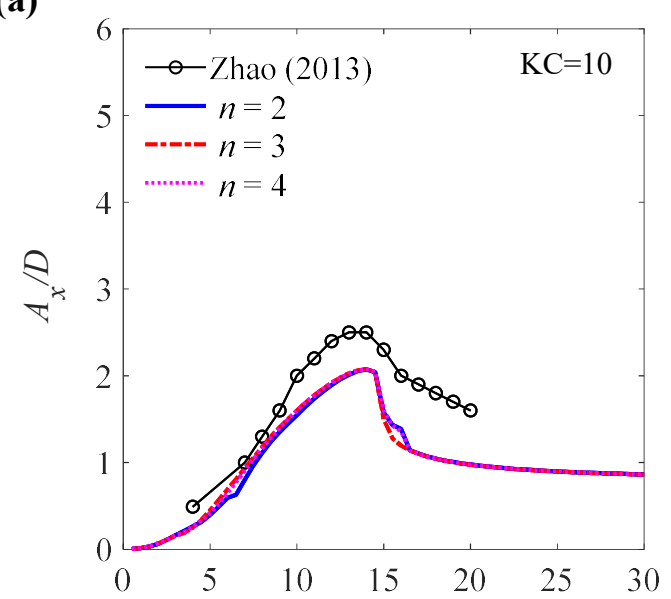

(c)

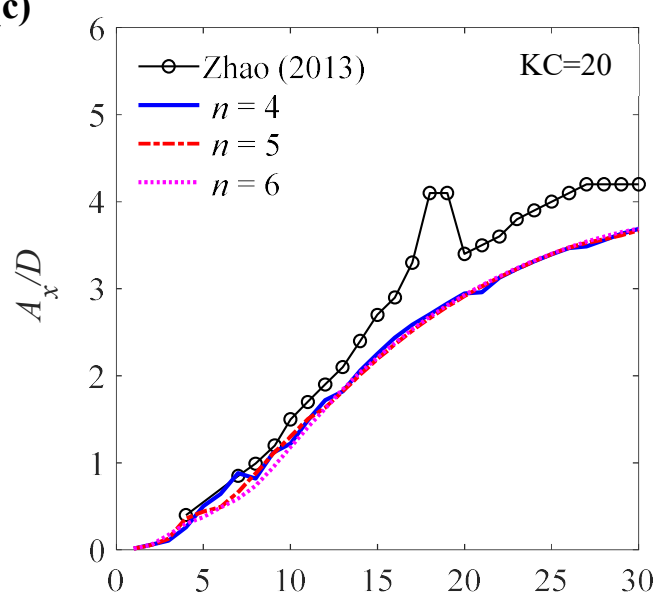

(e)

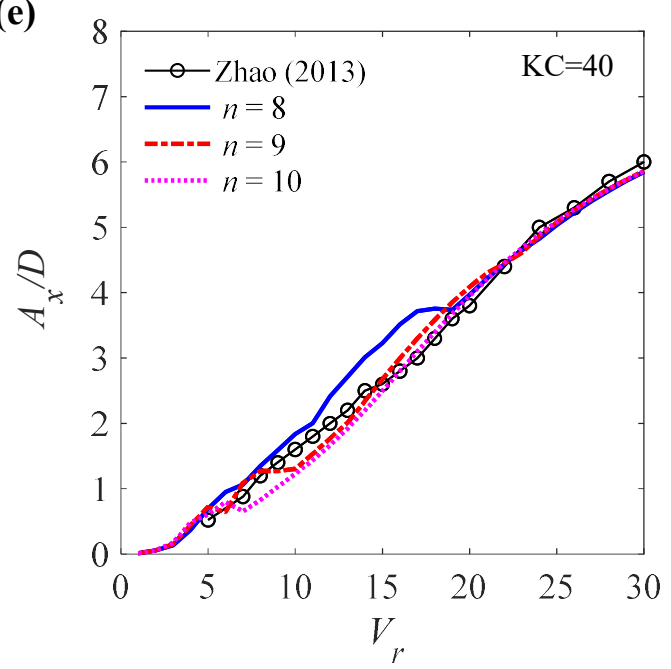

(b)

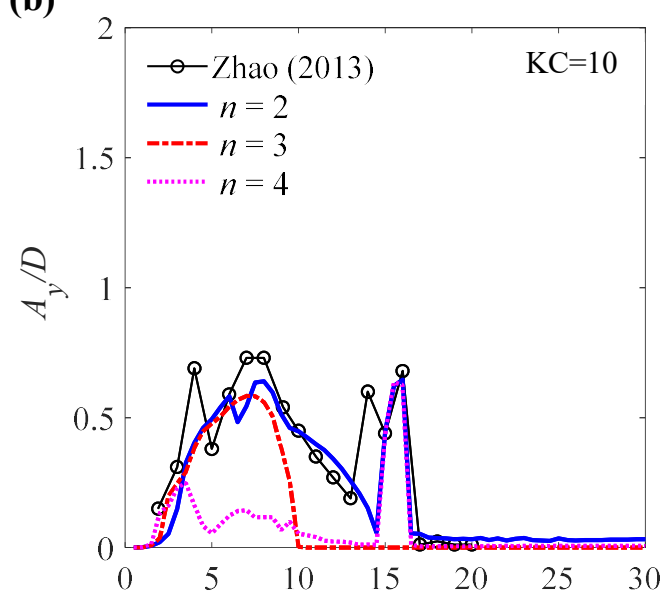

(d)

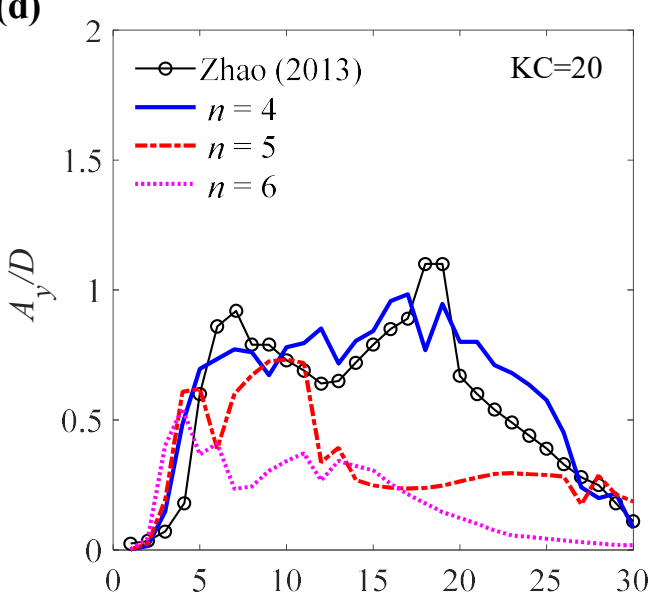

(f)

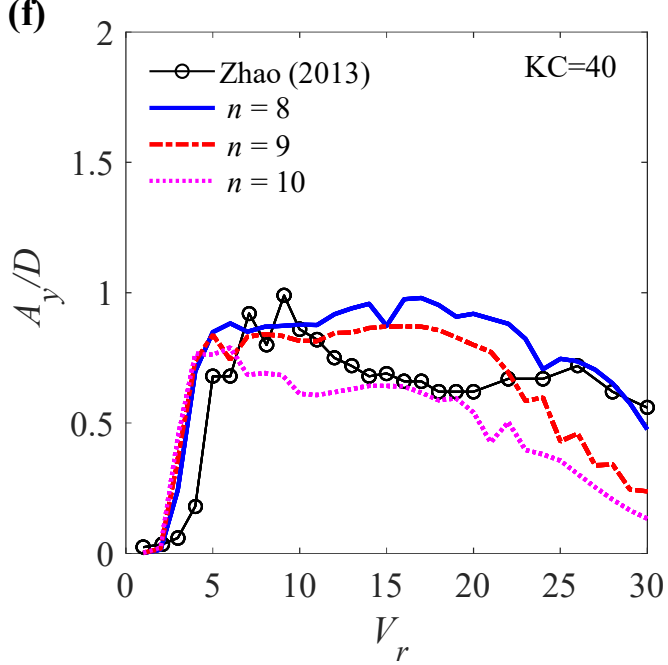

Figure 3. Comparisons of $A_{x} / D$ and $A_{y} / D$ predicted by wake oscillators with varying $n$ for different $\mathrm{KC}$ versus CFD results of Zhao (2013). 
(a)

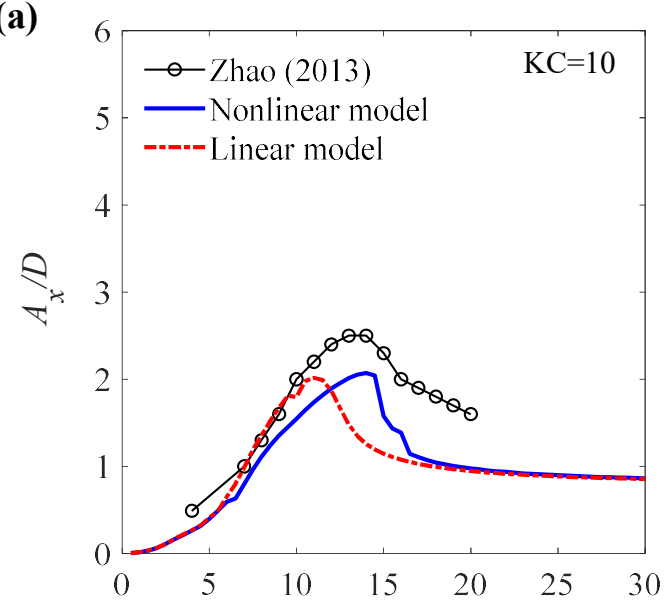

(c)

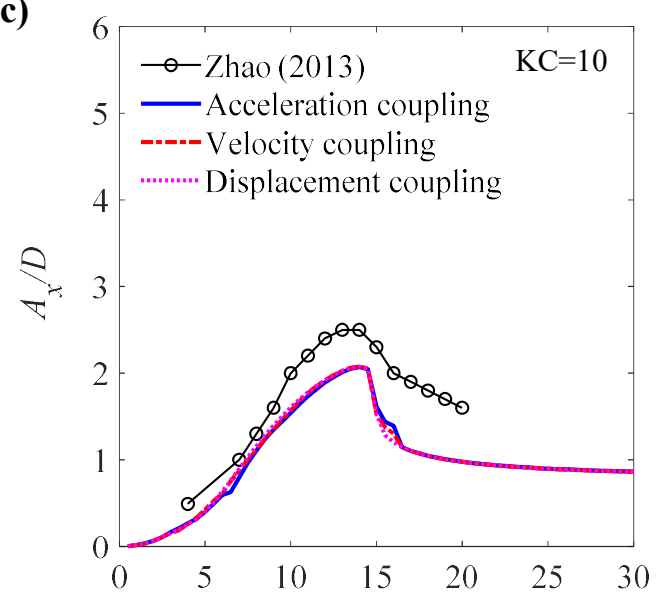

(e)

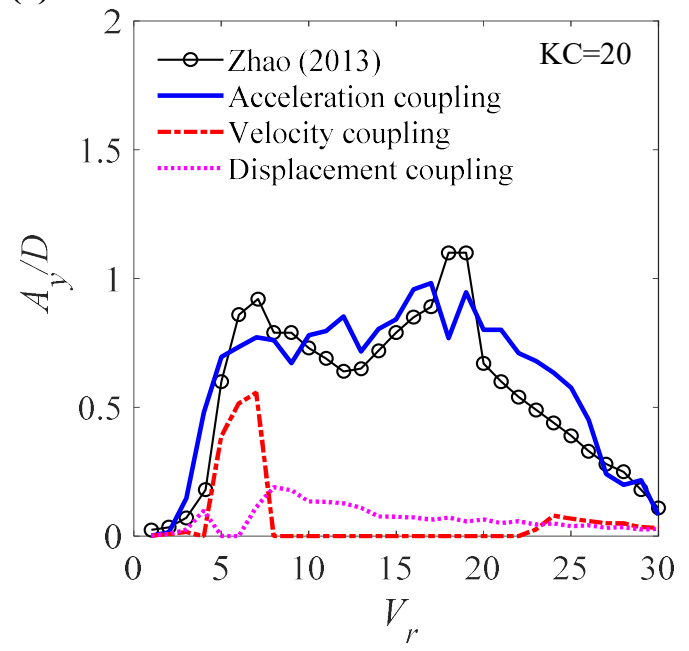

(b)

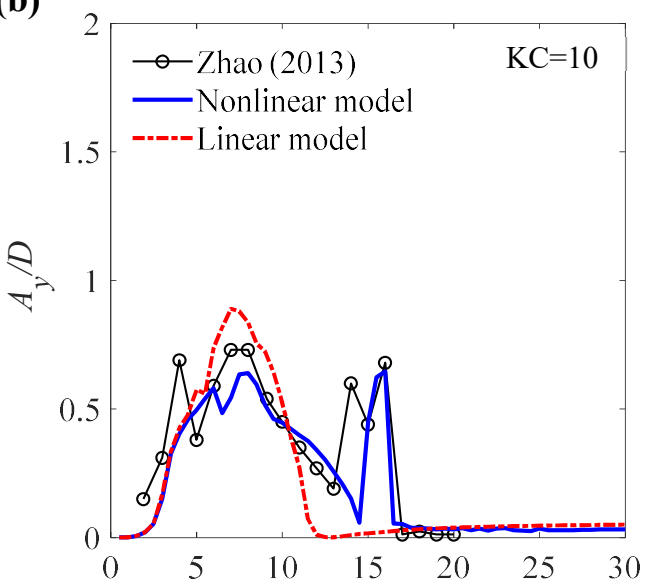

(d)

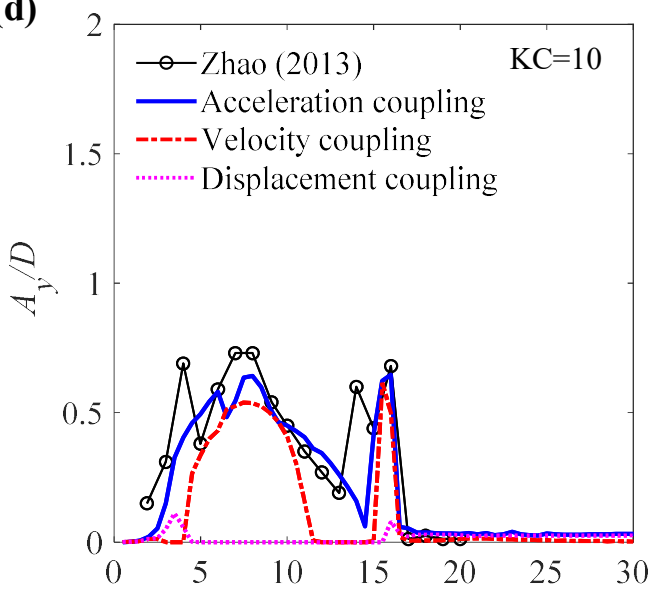

(f)

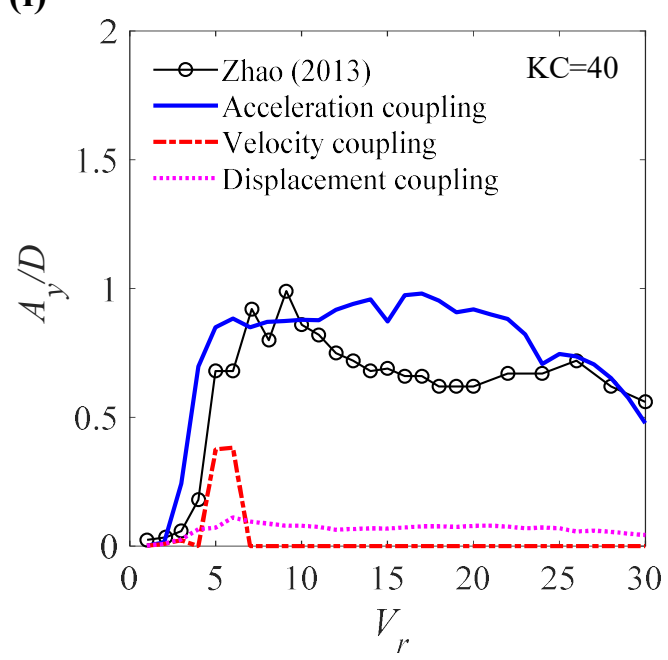

Figure 4. Comparisons of $A_{x} / D$ and $A_{y} / D$ predicted by wake oscillators for different $\mathrm{KC}$ versus CFD results of Zhao (2013): (a, b) cylinder linear vs. nonlinear stiffness models, (c-f) cylinder displacement, velocity vs. acceleration coupling terms in wake oscillators (Eqs. (12) and (14)). 
(a)

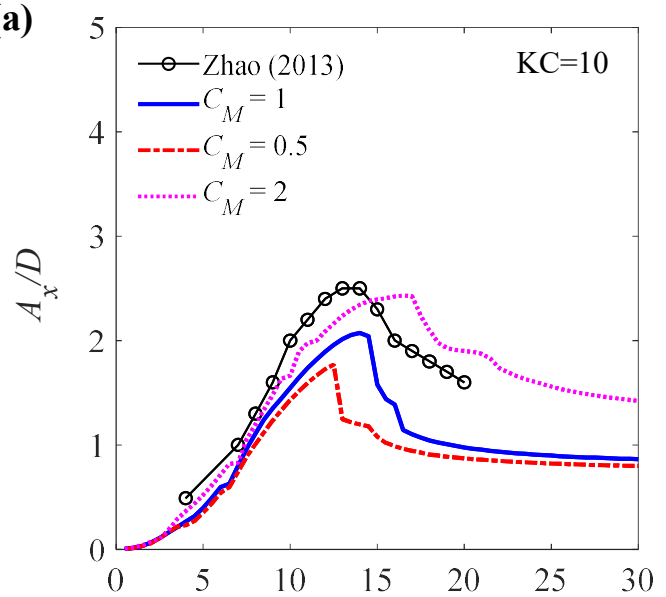

(c)

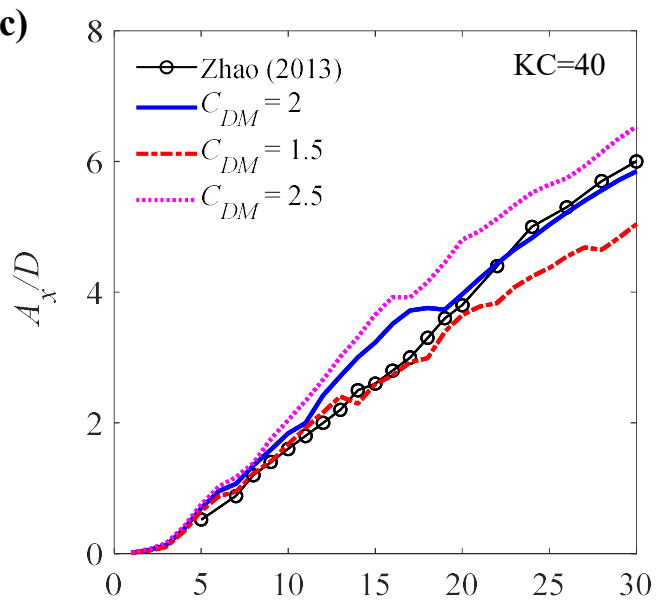

(e)

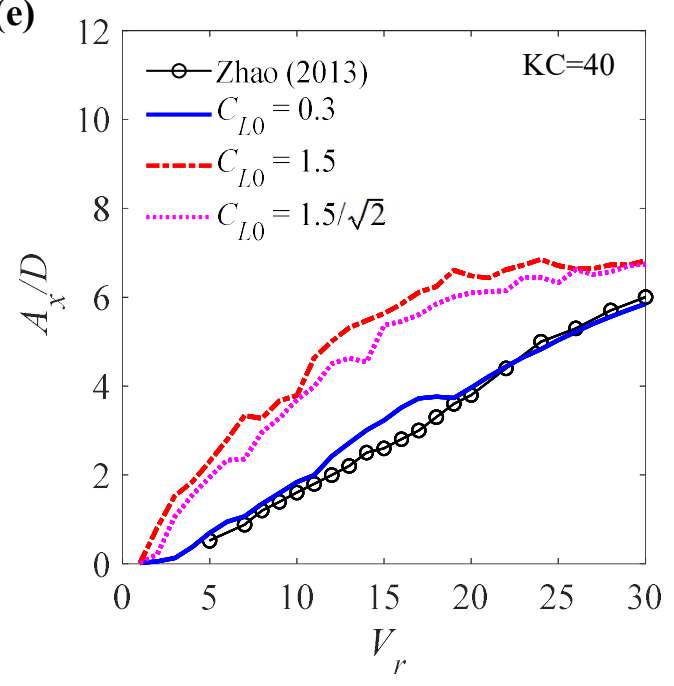

(b)

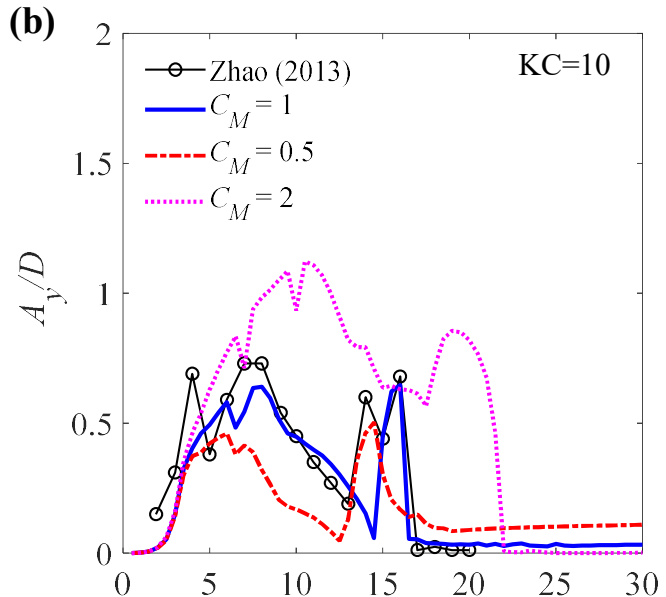

(d)

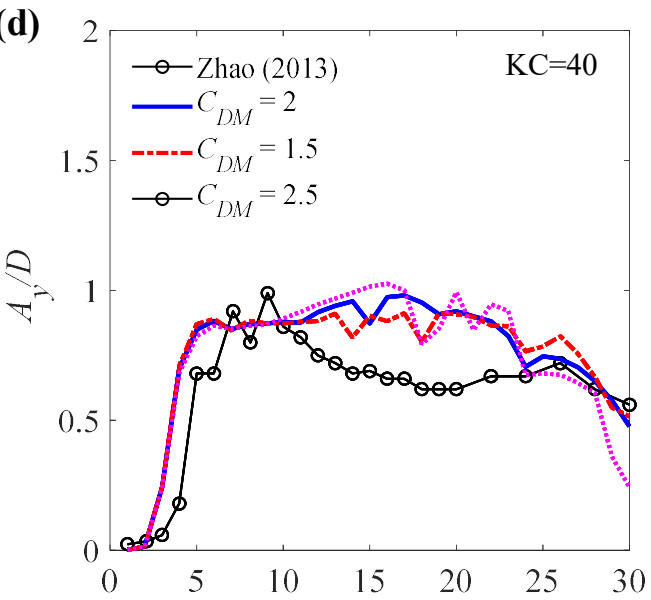

(f)

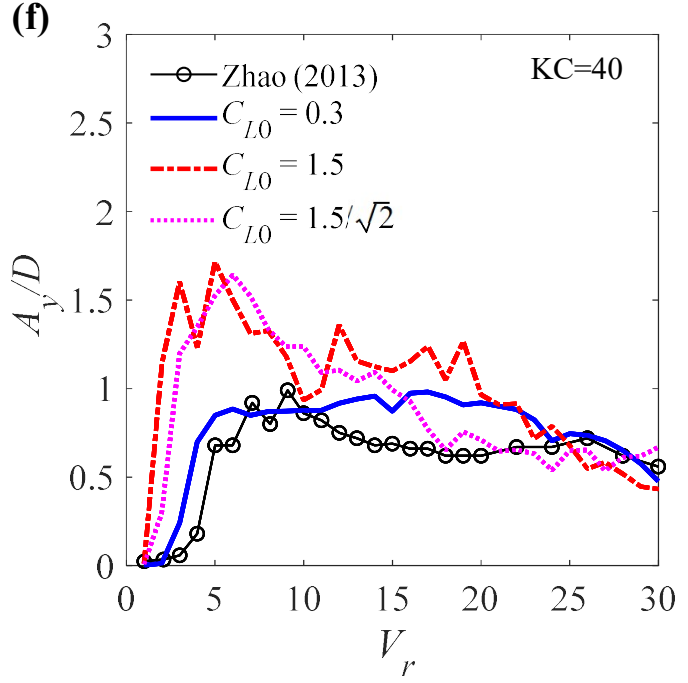

Figure 5. Comparisons of $A_{x} / D$ and $A_{y} / D$ predicted by wake oscillators for different $\mathrm{KC}$ versus CFD results of Zhao (2013), with effects of varying (a, b) $C_{M}$, (c, d) $C_{D M}$ and (e, f) $C_{L 0}$. 
(a)
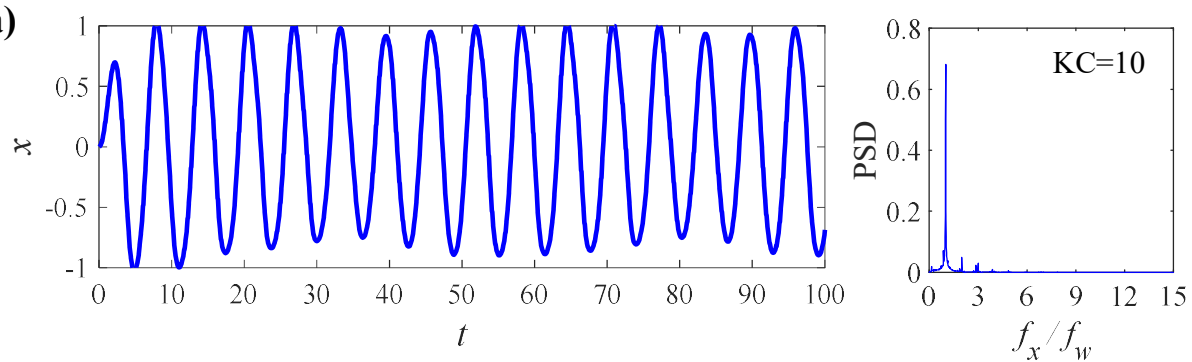

(b)
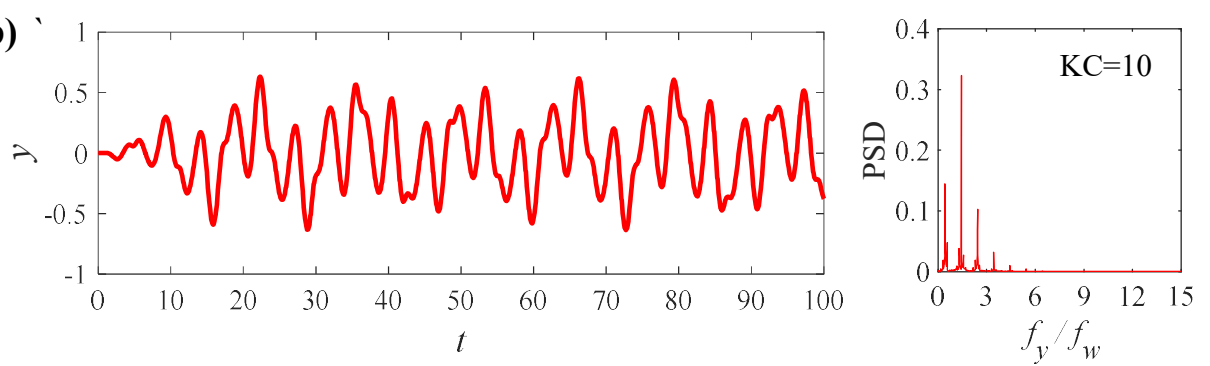

(c)
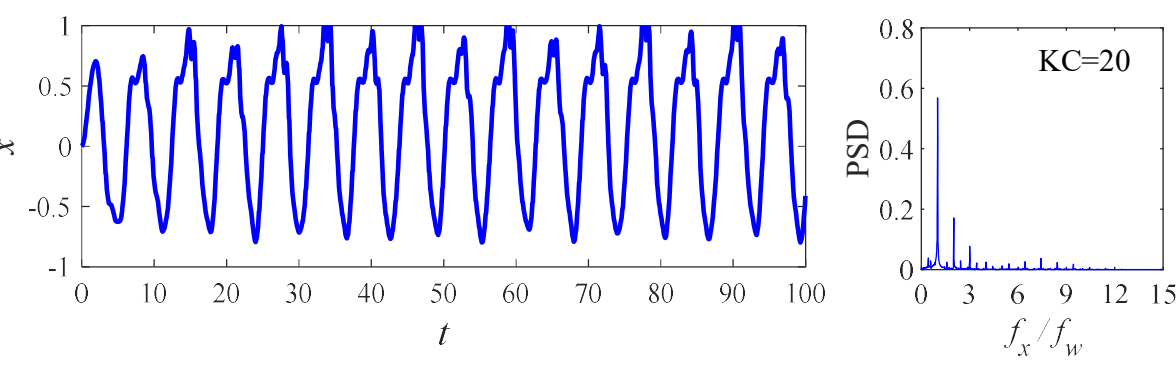

(d)
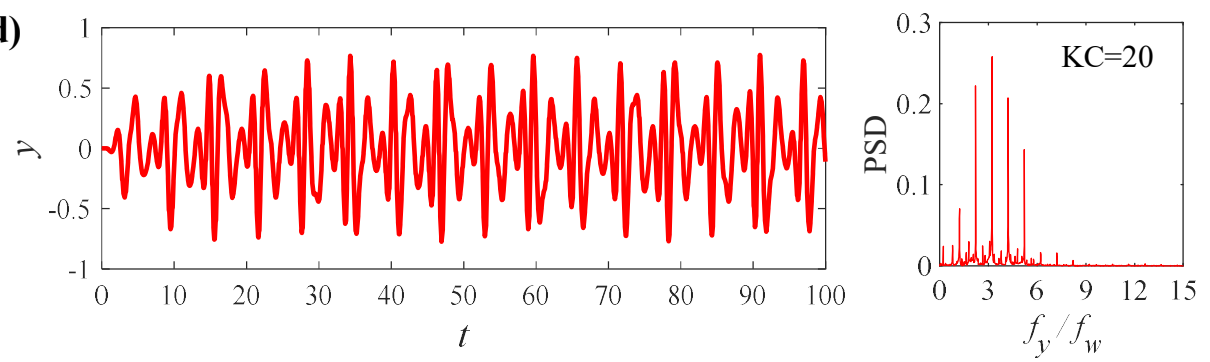

(e)
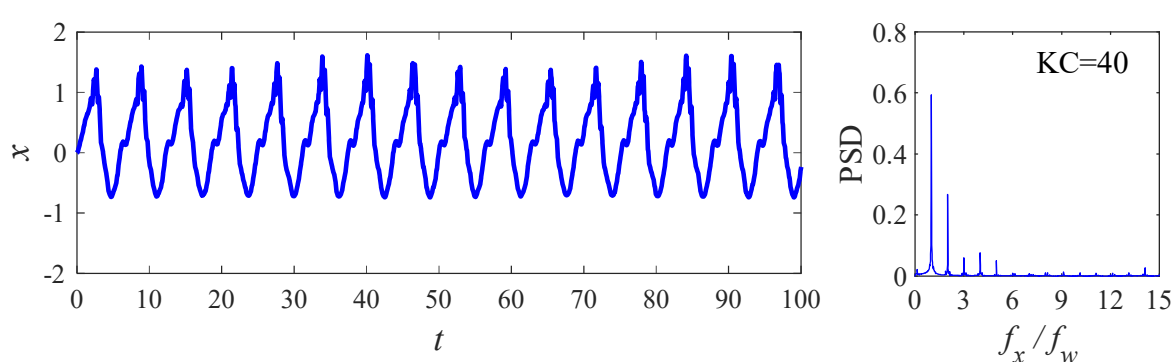

(f)
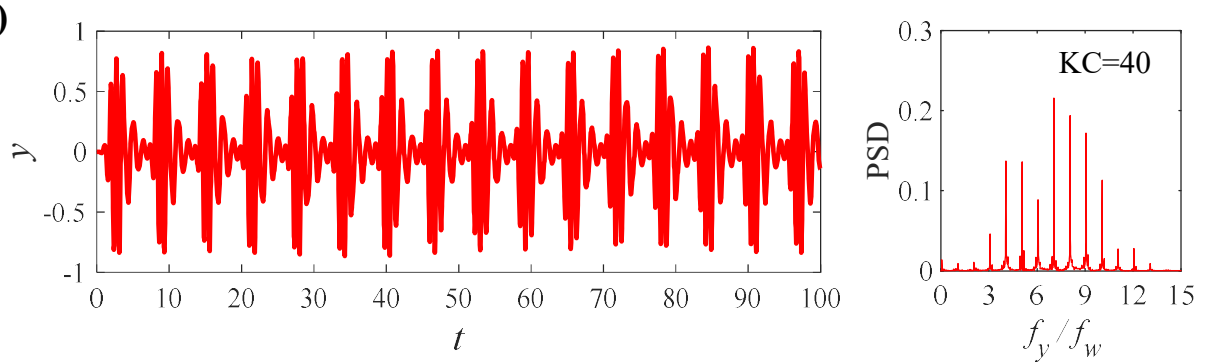

Figure 6. Illustrative in-line and cross-flow response time histories predicted by wake oscillators and associated FFT spectra at $V_{r}=7.5$ for different $\mathrm{KC}$. 
(a)

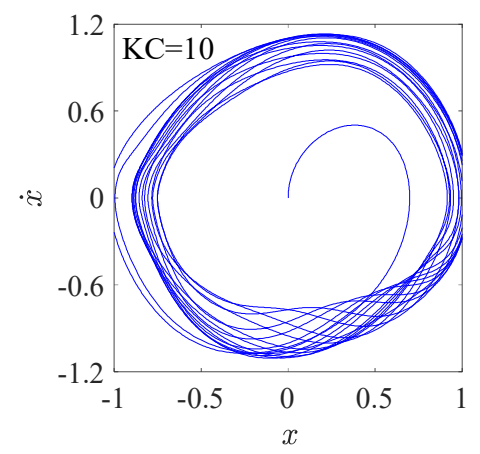

(d)

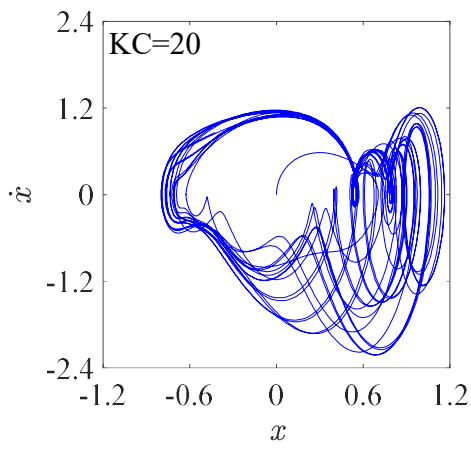

(g)

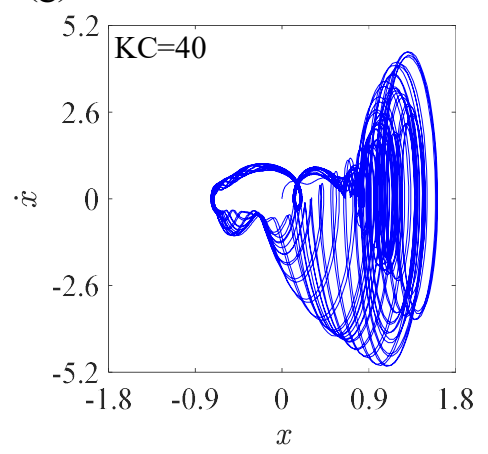

(b)

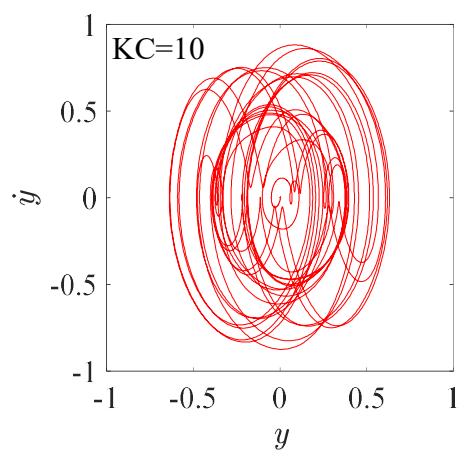

(e)

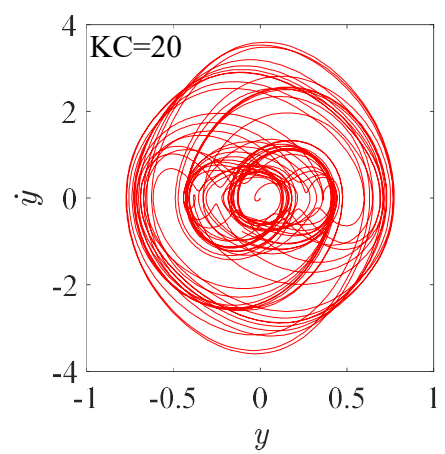

(h)

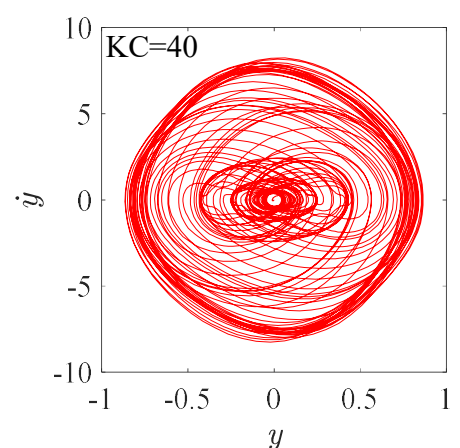

(c)

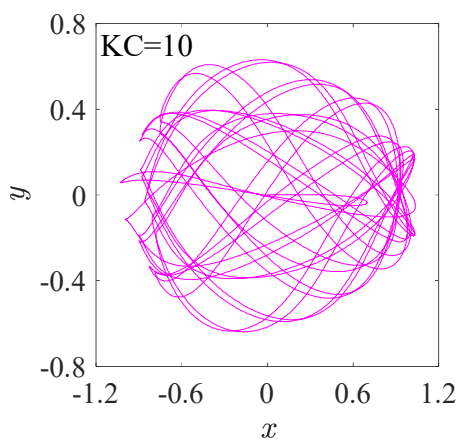

(f)

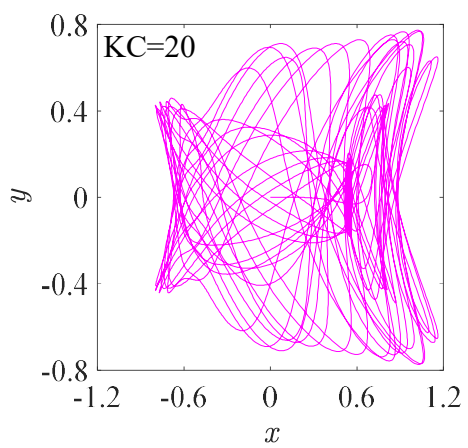

(i)

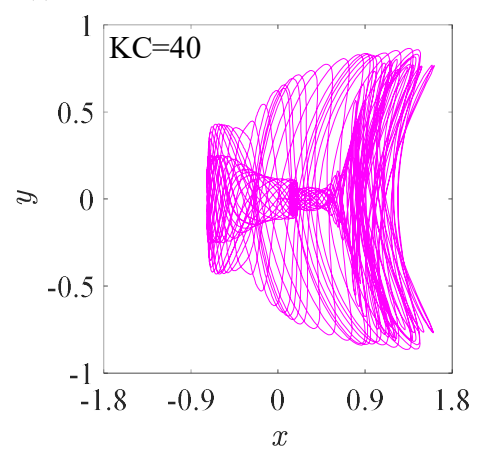

Figure 7. Illustrative in-line and cross-flow response phase portraits and $x-y$ trajectories predicted by wake oscillators, associated with Fig. 6 at $V_{r}=7.5$ for different KC. 


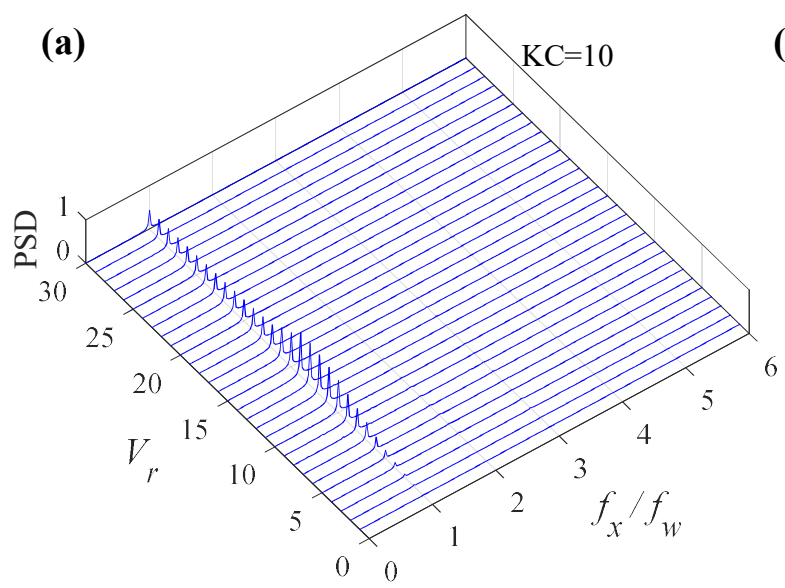

(b)
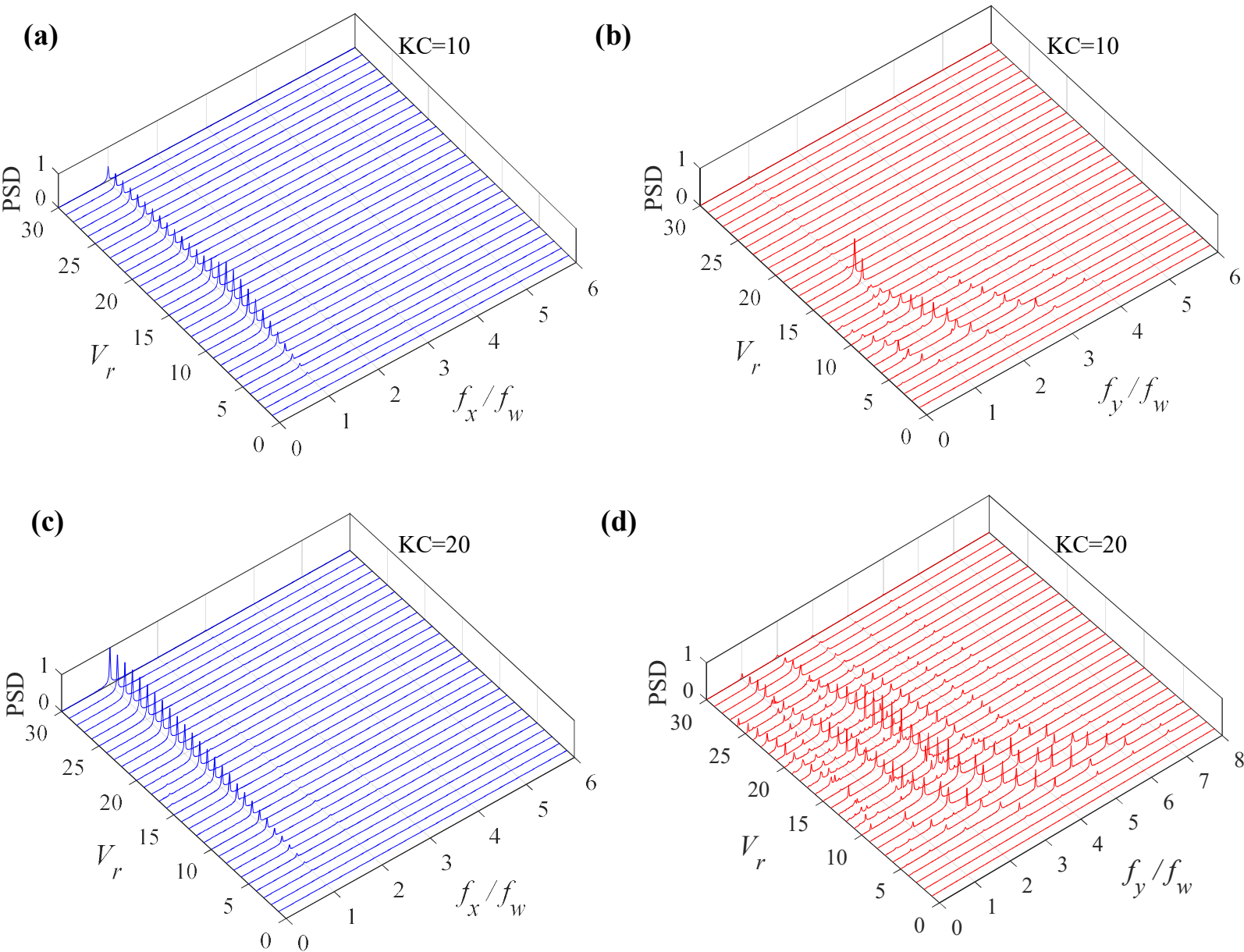

(d)
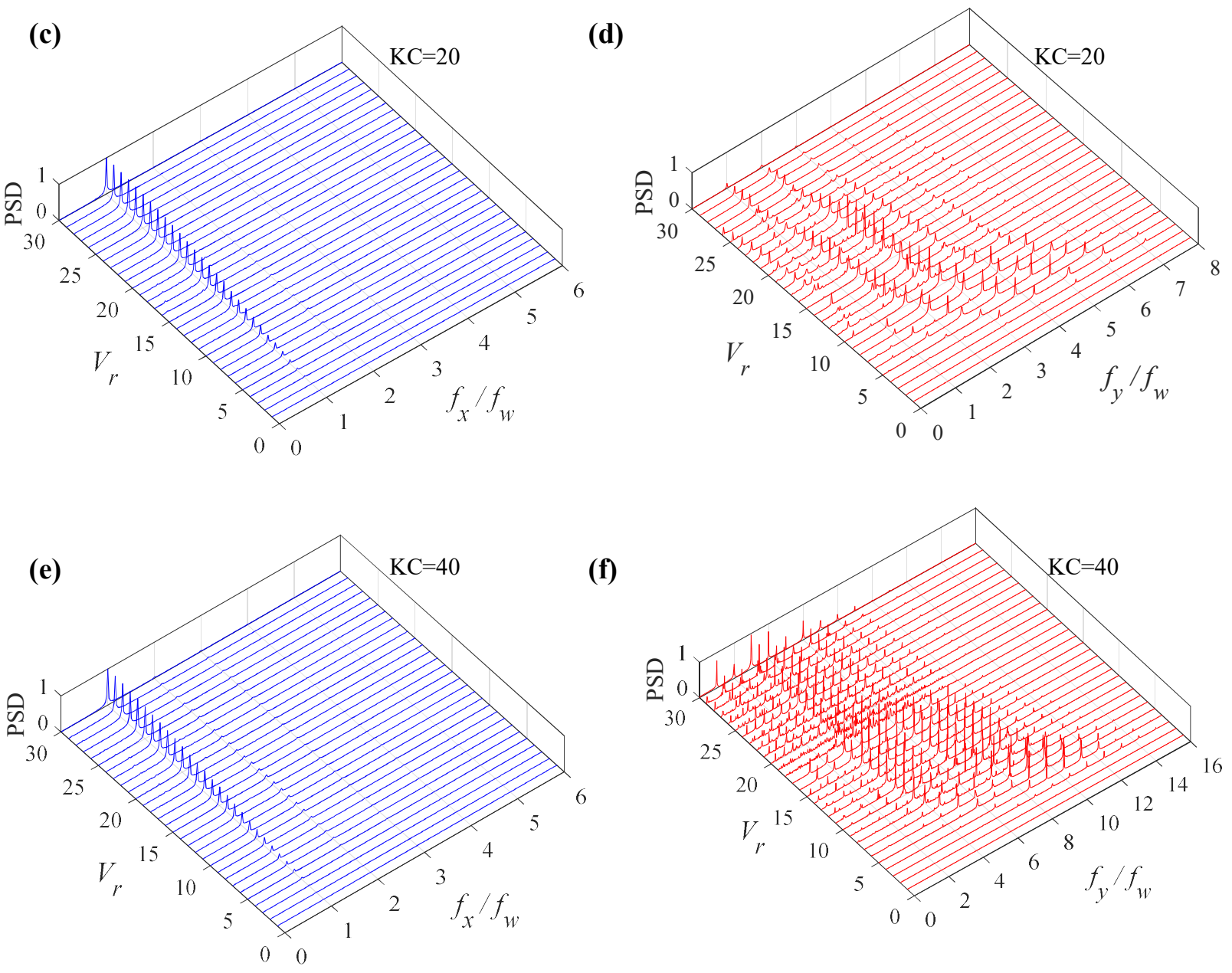

Figure 8. In-line and cross-flow oscillation frequency spectra predicted by wake oscillators in case of varying $V_{r}$ for different $\mathrm{KC}$. 
(a)

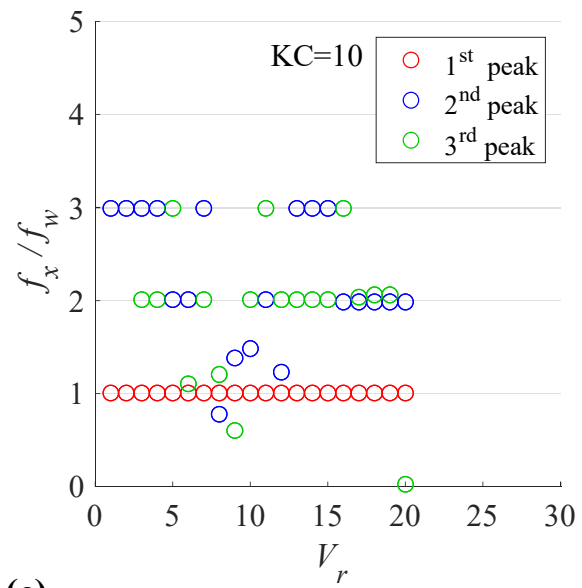

(c)

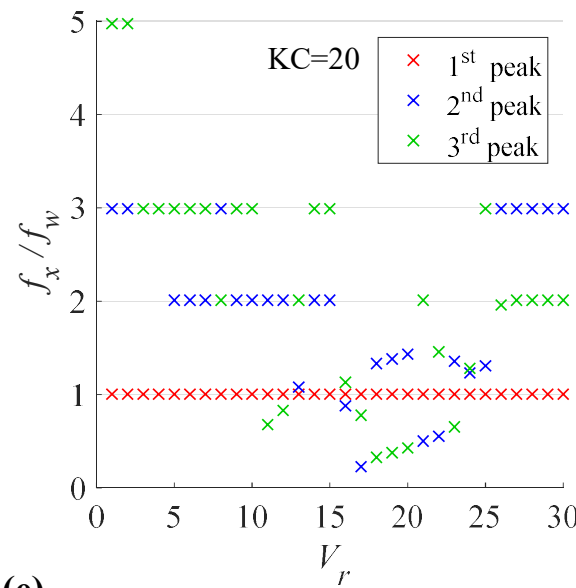

(e)

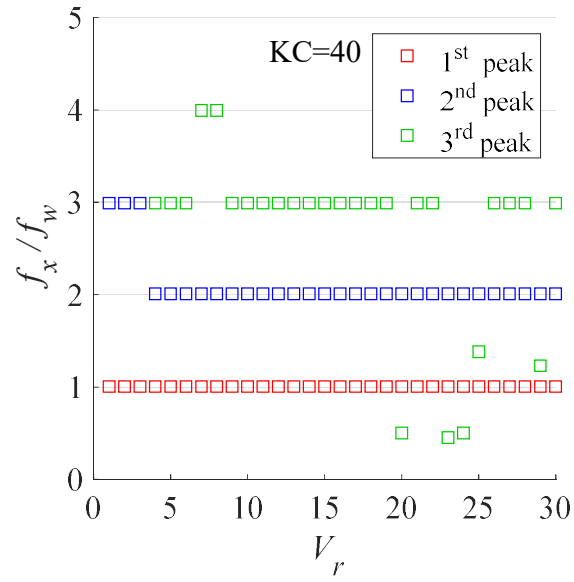

(b)

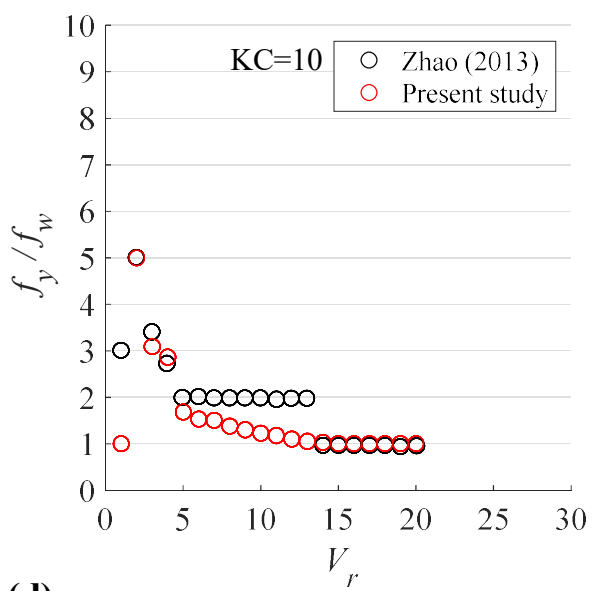

(d)

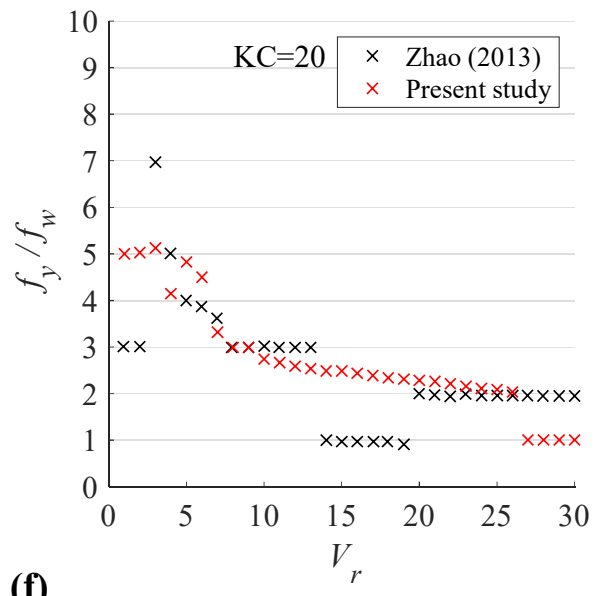

(f)

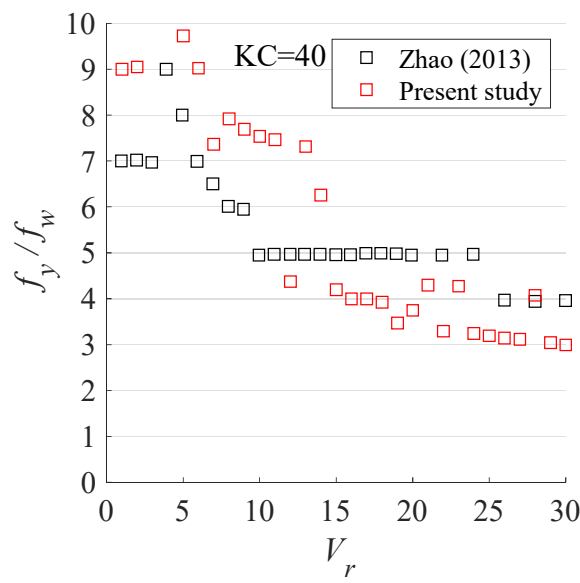

Figure 9. Extracted in-line (first three dominant) and cross-flow (primary) oscillation frequency components predicted by wake oscillators in the case of varying $V_{r}$ for different $\mathrm{KC}$, in comparison with CFD results (Zhao, 2013). 
(a)

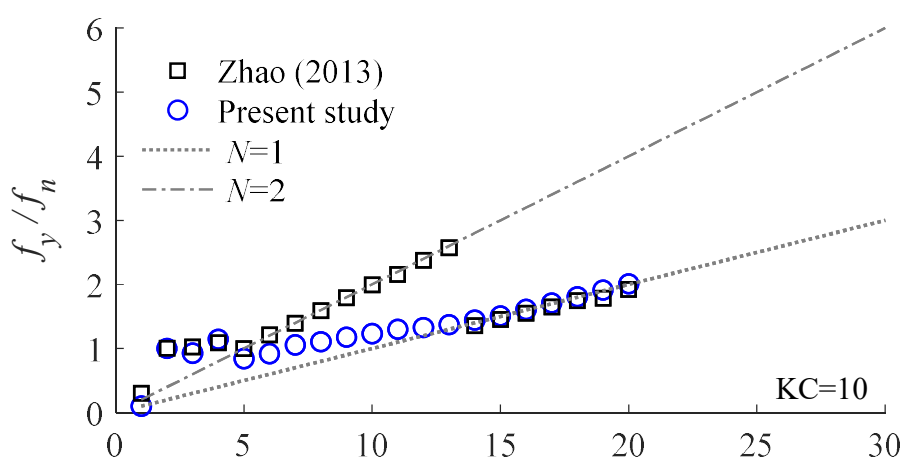

(b)

(c)
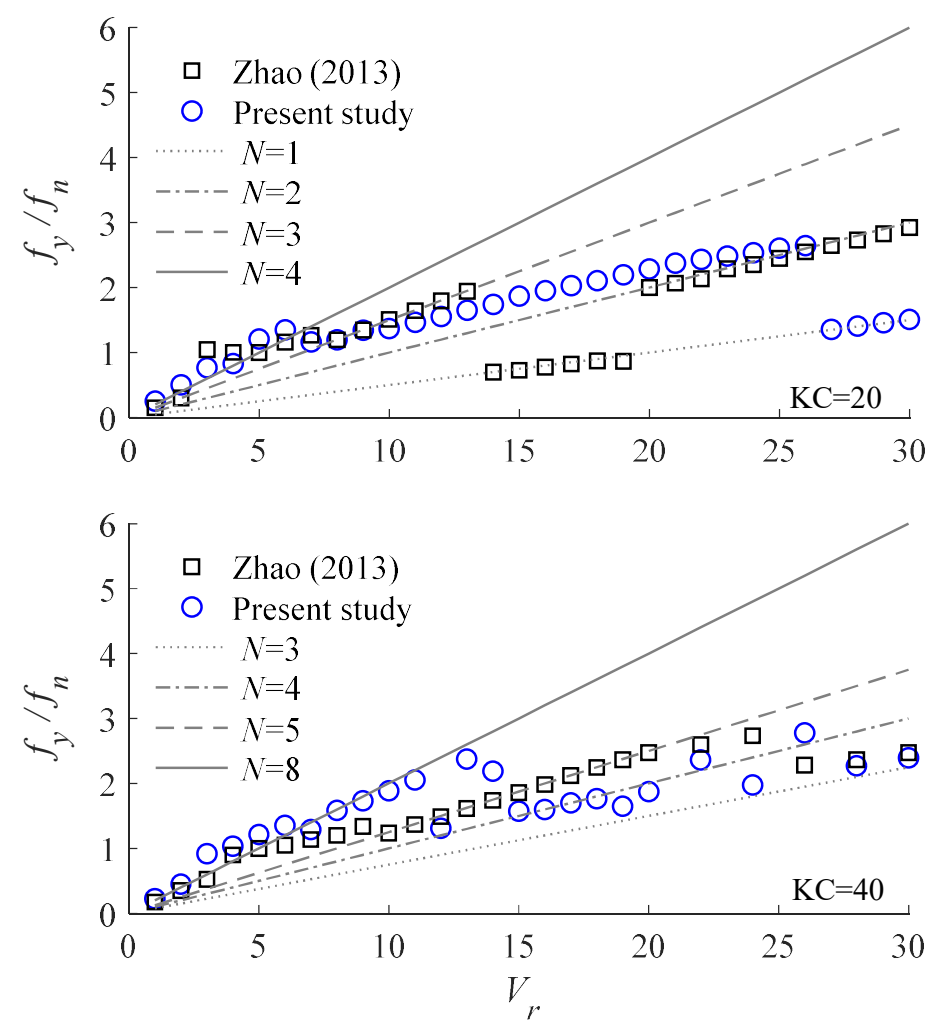

Figure 10. Dominant cross-flow oscillation frequencies normalized by natural frequency in comparison with CFD results of Zhao (2013) in the case of varying $V_{r}$ for different KC. Reference lines of $N$ integers are also plotted. 

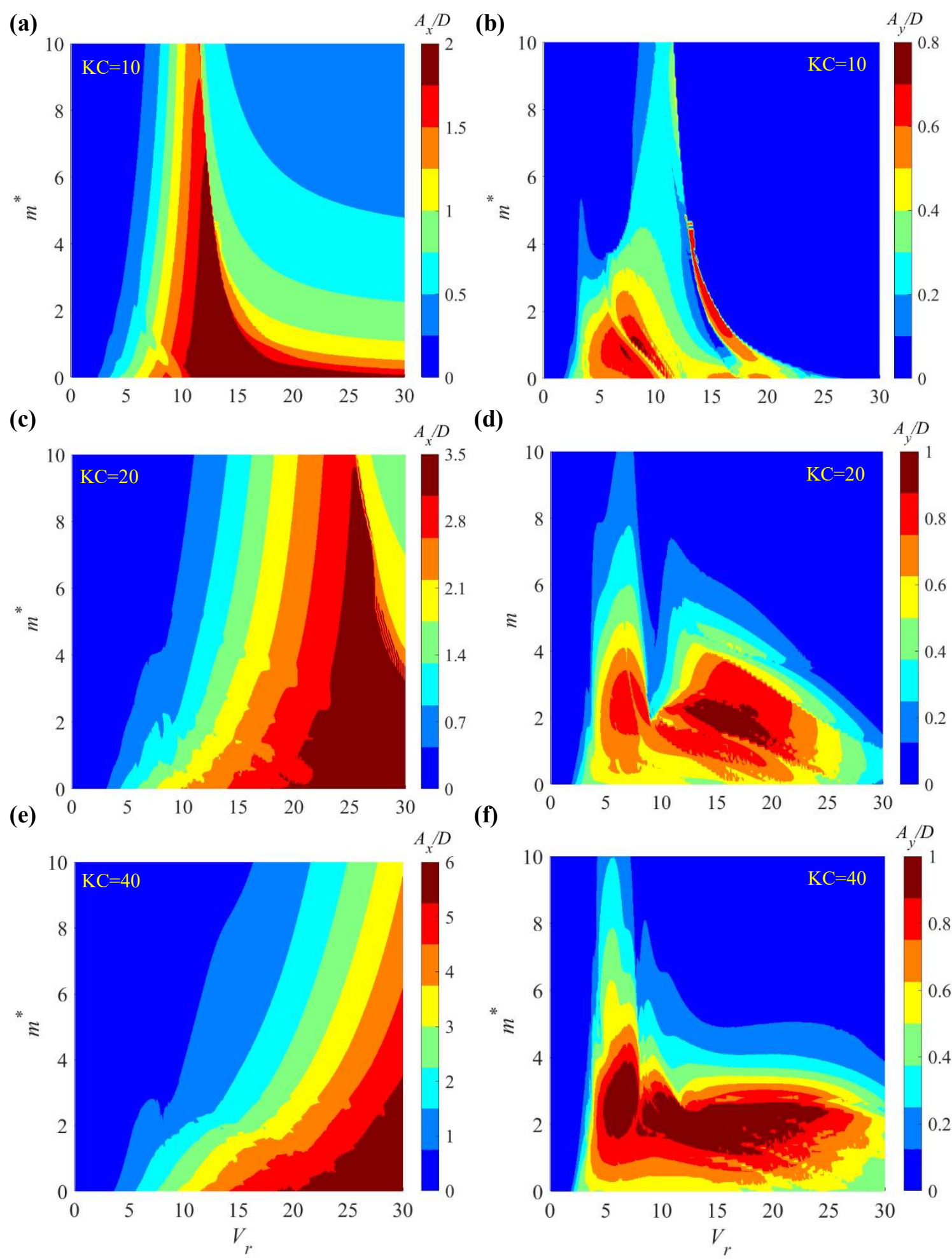

Figure 11. Contour plots of $A_{x} / D$ and $A_{y} / D$ as functions of $V_{r}$ and $m^{*}$ predicted by wake oscillators for a given $n=2,4$ and 8 for $\mathrm{KC}=10,20$ and 40, respectively. 


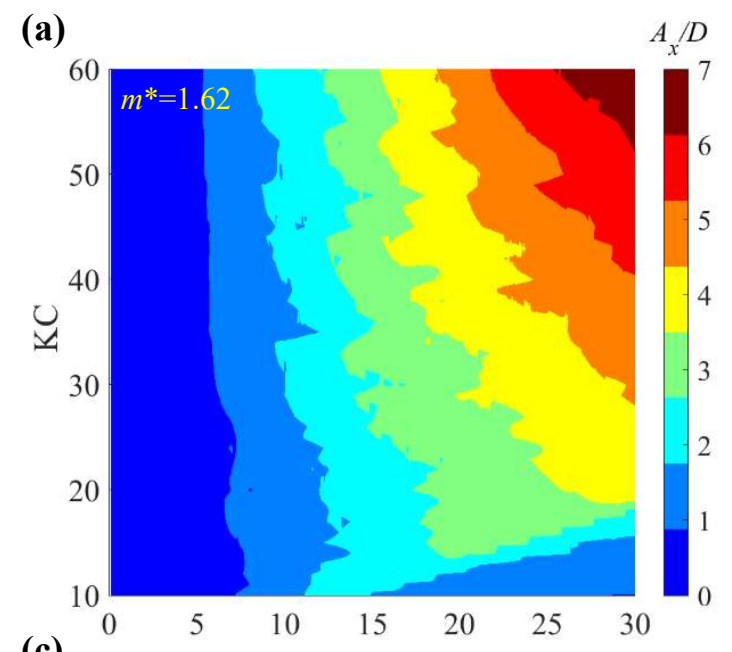

(c)

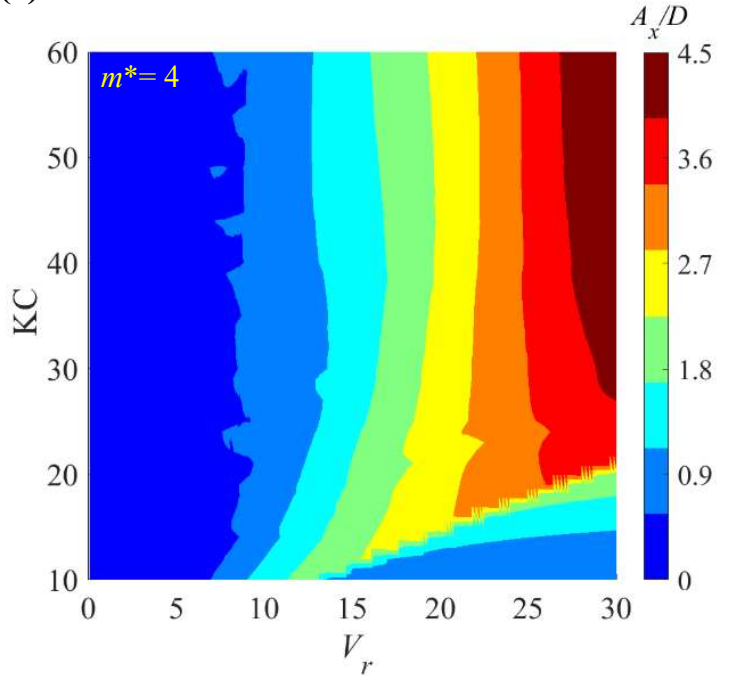

(b)

(d)
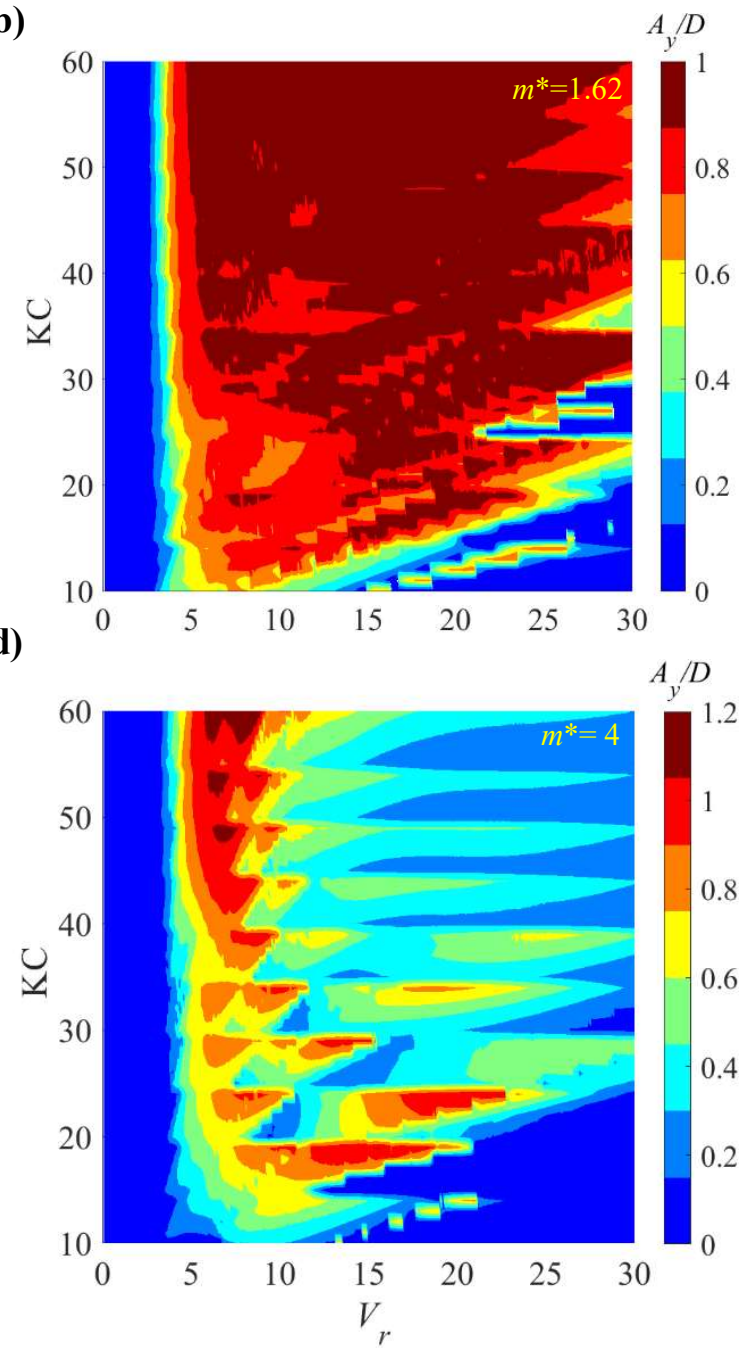

Figure 12. Contour plots of $A_{x} / D$ and $A_{y} / D$ as functions of $V_{r}$ and $\mathrm{KC}$ predicted by wake oscillators for a variable range of $n$ and for different $m^{*}$ cases. 


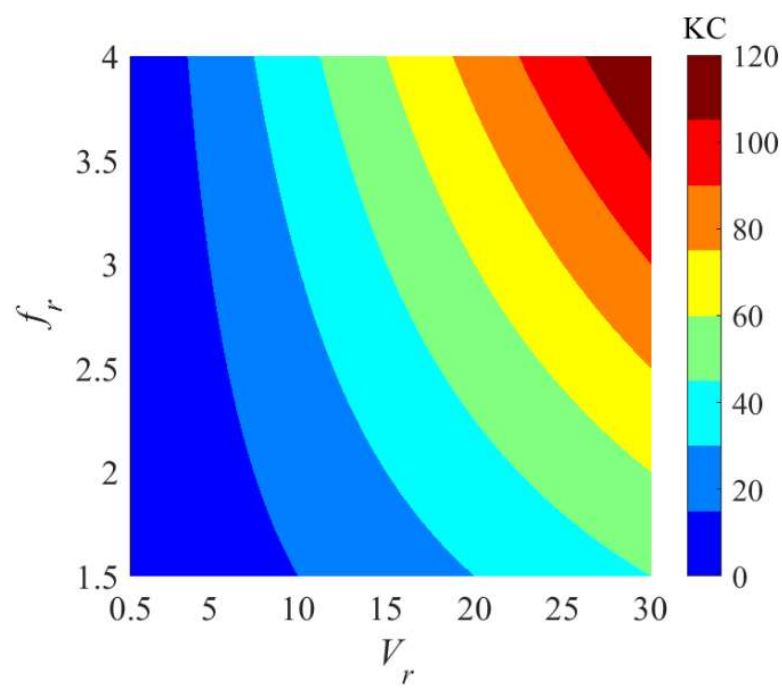

\begin{tabular}{|c|c|c|c|c|}
\hline \multirow{3}{*}{$m^{*}$} & \multirow{2}{*}{$f_{r}$} & \multicolumn{3}{|c|}{ Maximum cross-flow responses } \\
\cline { 2 - 5 } & & $V_{r}$ & $\mathrm{KC}$ & $A_{y} / D$ \\
\hline \multirow{4}{*}{1.62} & 1.5 & 20.5 & 31 & 0.99 \\
\cline { 2 - 5 } & 2 & 18.5 & 37 & 0.99 \\
\cline { 2 - 5 } & 2.5 & 19.0 & 48 & 1.00 \\
\cline { 2 - 5 } & 3 & 16.5 & 50 & 1.02 \\
\cline { 2 - 5 } & 3.5 & 19.0 & 67 & 0.99 \\
\cline { 2 - 5 } & 4 & 19.5 & 78 & 1.02 \\
\hline \multirow{4}{*}{4} & 1.5 & 9.5 & 14 & 0.66 \\
\cline { 2 - 5 } & 2 & 8.0 & 16 & 0.66 \\
\cline { 2 - 5 } & 2.5 & 7.5 & 19 & 0.64 \\
\cline { 2 - 5 } & 3 & 7.0 & 21 & 0.67 \\
\cline { 2 - 5 } & 3.5 & 6.5 & 23 & 0.68 \\
\cline { 2 - 5 } & 4 & 6.5 & 26 & 0.70 \\
\hline
\end{tabular}

Figure 13. Variations of $\mathrm{KC}$ as functions of $V_{r}$ and $f_{r}$, together with a summary of predicted maximum cross-flow responses, associated $V_{r}$ and $\mathrm{KC}$, extracted from Fig. 14 for different $m^{*}$ cases. 

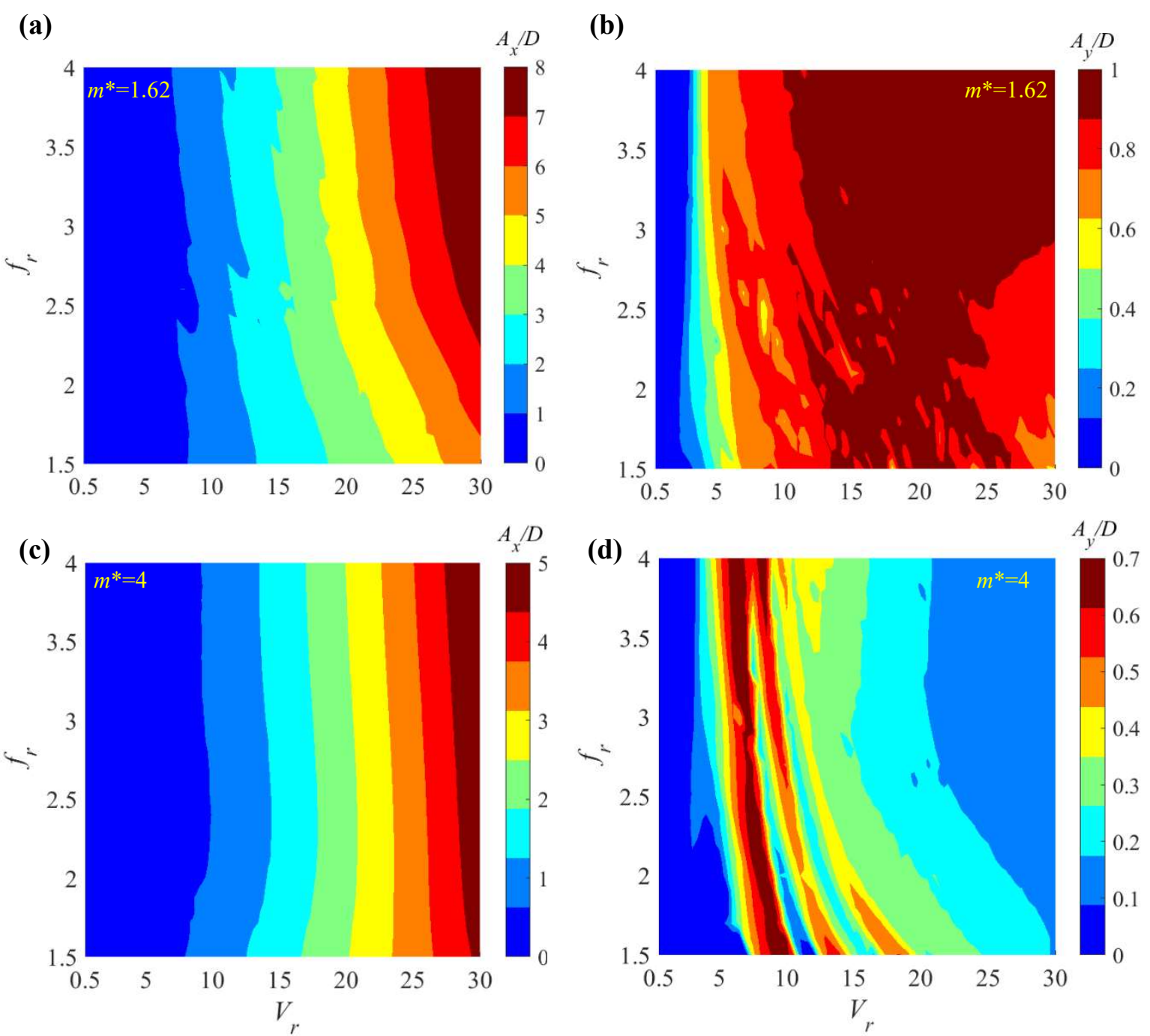

Figure 14. Contour plots of $A_{x} / D$ and $A_{y} / D$ as functions of $f_{r}$ and $V_{r}$ predicted by wake oscillators in the case of varying $\mathrm{KC}$ as in Fig. 13 for different $m^{*}$. 
(a)

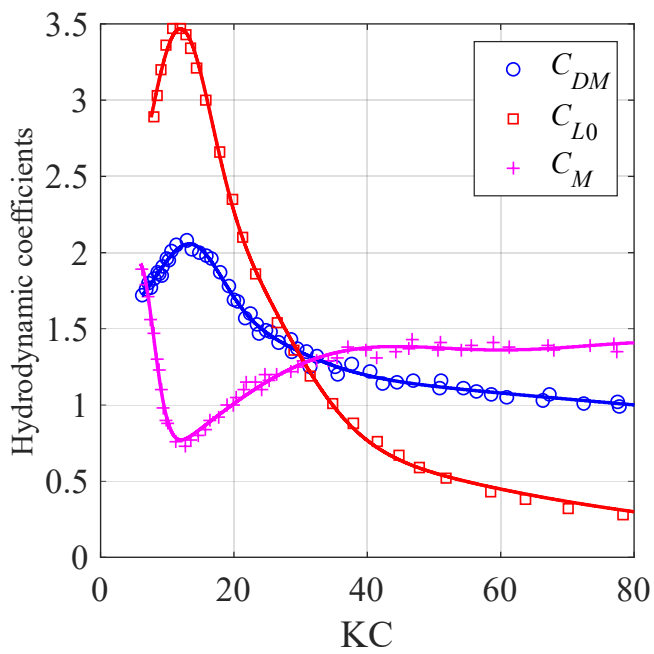

(c)

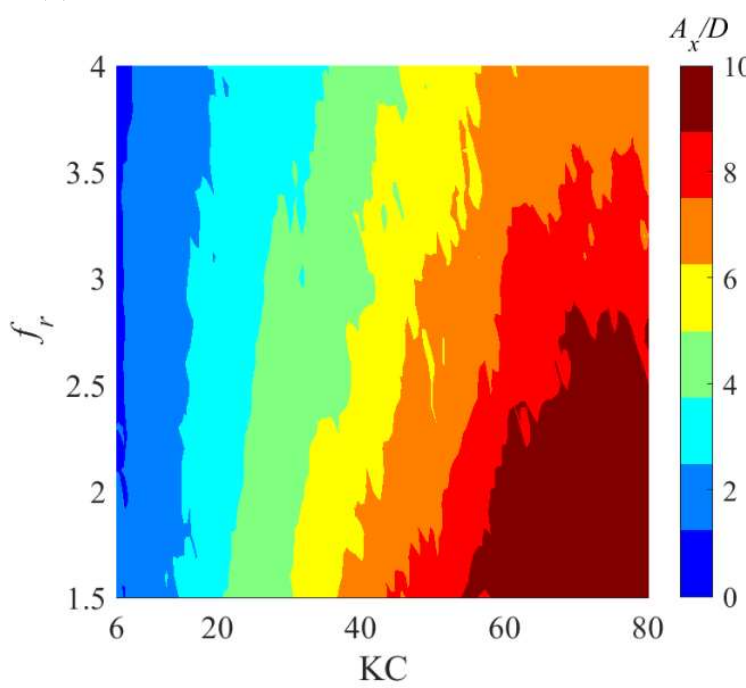

(b)

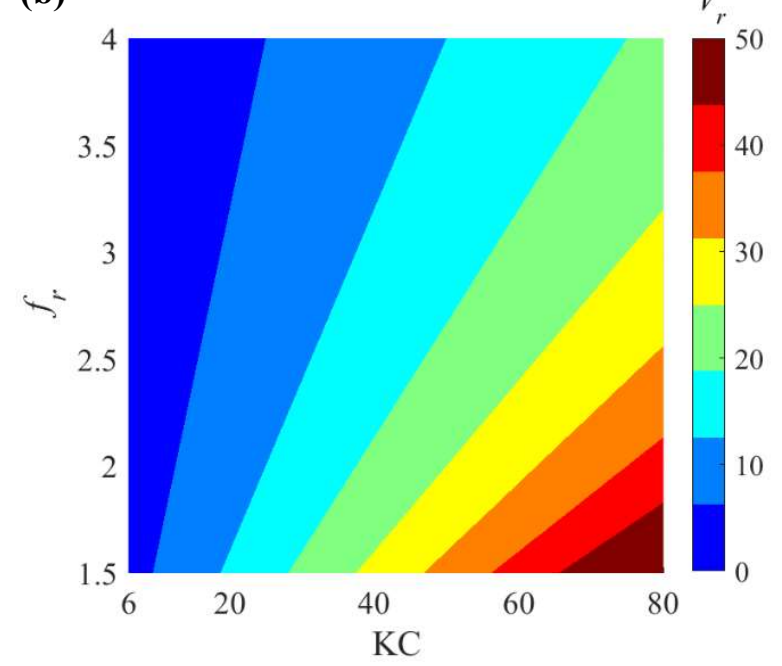

(d)

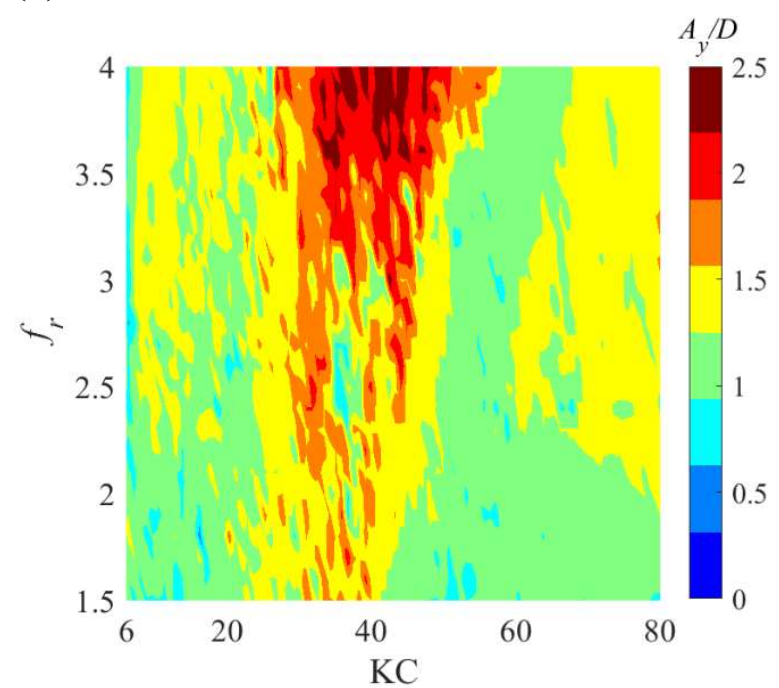

Figure 15. (a) Hydrodynamic coefficient data (symbols) and associated fitting curves (solid lines) as function of $\mathrm{KC}$ for a given $\beta=\mathrm{Re} / \mathrm{KC}=1107$, (b) variations of $V_{r}$ as function of $f_{r}$ and $\mathrm{KC}$, and associated contour plots of (c) $A_{x} / D$ and (d) $A_{y} / D$ predicted by wake oscillators for $m^{*}=1.62$. 
(a)

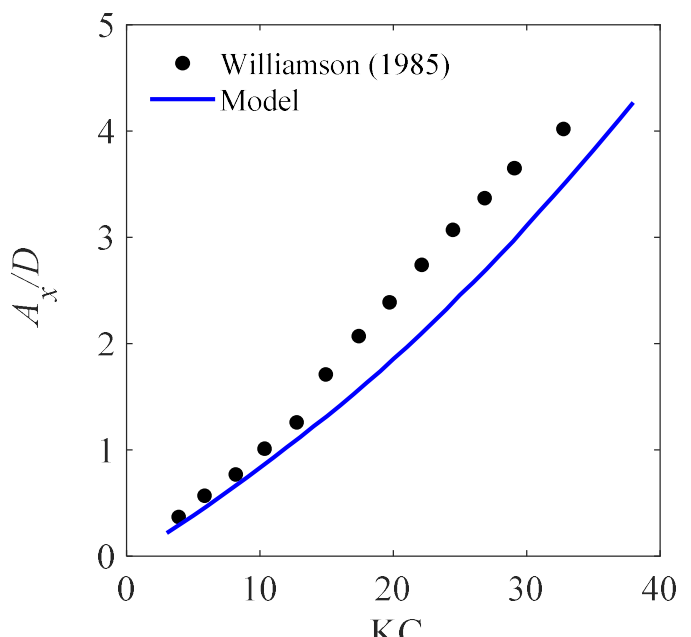

(c)

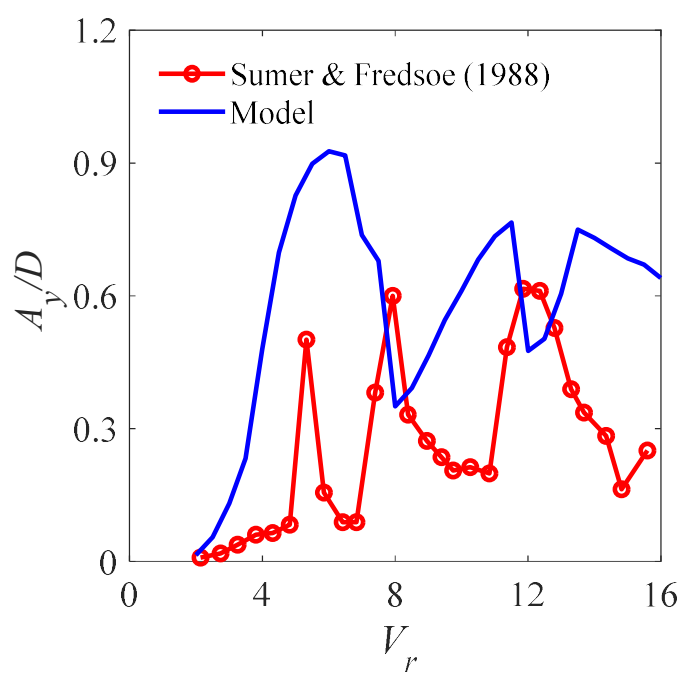

(b)
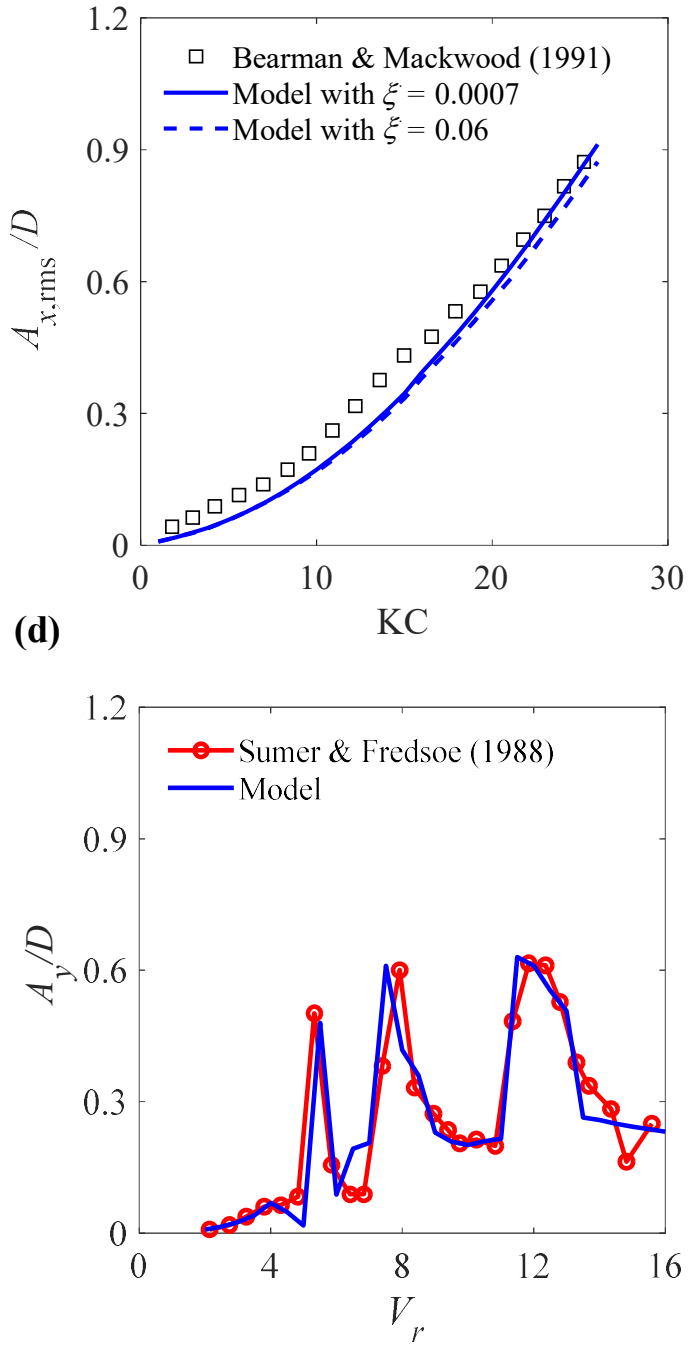

Figure 16. Comparisons of 1-DOF $(a, b)$ in-line and $(c, d)$ cross-flow VIV predictions by wakeoscillators versus experimental results in the literature. Results in (a)-(c) are based on empirical coefficients deduced from 2-DOF VIV model whereas results in (d) are based on new best-fit $\Lambda_{y}$ values for 1-DOF VIV model. 